\title{
The identification of the species of the 'Spilogona contractifrons species-group' and the 'Spilogona nitidicauda species-group' (Diptera, Muscidae) based on morphological and molecular analysis
}

\author{
Vera S. SOROKINA ${ }^{1, *} \&$ Elena V. SHAIKEVICH ${ }^{2}$ \\ ${ }^{1}$ Siberian Zoological Museum, Institute of Systematics and Ecology of Animals, Russian Academy of \\ Sciences, Siberian Branch, Frunze str. 11, Novosibirsk 630091, Russia. \\ ${ }^{2}$ Laboratory of Insect Genetics, N.I. Vavilov Institute of General Genetics, Russian Academy of \\ Sciences, Gubkin Street 3, Moscow 119991, Russia. \\ "Corresponding author: sorokinavs@mail.ru \\ ${ }^{2}$ Email: elenashaikevich@mail.ru \\ ${ }^{1}$ urn:lsid:zoobank.org:author:394AB897-8128-49F4-806D-4DF36557C48A \\ ${ }^{2}$ urn:lsid:zoobank.org:author:39F9E7C8-6761-46C0-BEDE-81F396FBD5AC
}

\begin{abstract}
Muscid species of the 'Spilogona contractifrons species-group' (Spilogona alticola (Malloch, 1920), S. arctica (Zetterstedt, 1838), S. contractifrons (Zetterstedt, 1838), S. orthosurstyla Xue \& Tian, 1988 ) and of the 'Spilogona nitidicauda species-group' (S. nitidicauda (Schnabl, 1911), S. hissarensis Hennig, 1959, S. imitatrix (Malloch, 1921), S. platyfrons Sorokina, 2018) are notoriously difficult to distinguish. In this paper, their morphological features are analysed, images of the male head, frons and abdomen of all the species are given, and the male terminalia are figured. The study of extensive material has shown that all the morphologically recognised species in each of these groups are valid species. An identification key is provided for both groups of species. To confirm the morphological differences, genetic differences in the cytochrome oxidase I gene of flies of the 'Spilogona contractifrons speciesgroup' and of the 'Spilogona nitidicauda species-group' were analysed. It is shown that members of both groups of species have not only distinguishing morphological characters but also fixed substitutions in the DNA sequences. Since a low interspecific polymorphism is known in the Muscidae Latreille, 1802, the revealed genetic distances confirm the existence of separate species or subspecies in each of the groups studied.
\end{abstract}

Keywords. COI gene, DNA barcoding, flies, identification key, male terminalia.

Sorokina V.S. \& Shaikevich E.V. 2018. The identification of the species of the 'Spilogona contractifrons speciesgroup' and the 'Spilogona nitidicauda species-group' (Diptera, Muscidae) based on morphological and molecular analysis. European Journal of Taxonomy 484: 1-26. https://doi.org/10.5852/ejt.2018.484

\section{Introduction}

The genus Spilogona Schnabl, 1911 is one of the most speciose genera of the Muscidae Latreille, 1802 and is dominant both in the number of species and in the number of individuals in cold habitats, such 
as the high-mountain and latitudinal tundra (Sorokina 2012a, 2012b, 2013a, 2013b, 2017; Sorokina \& Khruleva 2012).

This genus has been most comprehensively studied in the Nearctic Region, in particular in the territory of northern Canada and Alaska (Huckett 1965). The number of arctic species of Spilogona known from the Nearctic Region is 88. The tundra zones of the Palaearctic Region have not been studied for a long time, and accordingly the number of known species of Spilogona for these territories is significantly less (66) (Hennig 1959a, 1959b, 1959c). Recent papers on this genus have increased the number of Palaearctic species to 90, whilst the number of Nearctic species has decreased to 86 (Sorokina 2018).

It should be noted that the changes in the number of species of Spilogona is due not only to the description of new species, but also to the synonymization of some Nearctic and Palaearctic species. The reason for the latter is that most species of Spilogona have a circumpolar or arctic-montane distribution, but this fact was not previously taken into account by researchers when describing new species. Moreover, the genus Spilogona consists of small to medium-sized, dark-coloured muscids covered in greyish to blackish-brown dusting, frequently revealing paired dark markings, which makes them very similar to each other. Furthermore, the high number of species encountered in arctic situations in combination with an often poor state of preservation of specimens collected in traps, preserved in alcohol, etc., make species identification of samples of Spilogona a difficult task even for the specialist. There are therefore still are many problems in clarifying the taxonomic status of the various known species of Spilogona.

This paper is dedicated to flies of the 'Spilogona contractifrons species-group' and the 'Spilogona nitidicauda species-group'. Members of these species groups are some of the most dominant in the tundra zone. Like other species of the genus, adults of these groups of species are predaceous on other small and soft-bodied insects close to running water. One of their important roles in nature is their known activity as predators on the adults and larvae of biting black flies (Simuliidae) and on other small insects (Werner \& Pont 2006a, 2006b). However, adults of these species live at the same time and in the same biotopes and have very similar morphological characters, and this causes difficulty with their identification and therefore confusion in the results of field research. Moreover, there are different opinions as to the number of existing species in each of these groups of species.

The 'Spilogona contractifrons species-group' includes four morphologically very similar species: S. contractifrons (Zetterstedt, 1838), S. alticola (Malloch, 1920), S. arctica (Zetterstedt, 1838) and S. orthosurstyla Xue \& Tian, 1988. The first three species are widely distributed, and consequently have given rise to discussion for many years. Opinions have varied about the presence of only two, or even one species, but not three. Collin (1930) recorded just two species, S. contractifrons and S. arctica from Greenland. Hennig (1959a: 288) and Pont (1986: 166) regarded S. arctica as a synonym of S. contractifrons. Spilogona alticola was previously known only from North America. In his monograph, Huckett (1965: 238) included all three as valid species but added, under the entry for S. contractifrons, "I am doubtful whether alticola Malloch can be regarded specifically as different to contractifrons". Later, Michelsen (2006: 118) reinstated S. arctica as a valid species. In a recent paper by the first author of the present paper, four species of this group of species were reported in the Altai Mountains (Sorokina 2018).

The 'Spilogona nitidicauda species-group' included three morphologically similar species: S. nitidicauda (Schnabl, 1911), S. hissarensis Hennig, 1959 and S. imitatrix (Malloch, 1921). Recently, a fourth species (S. platyfrons Sorokina, 2018) of this group has been described (Sorokina 2018). Spilogona hissarensis is known only from the type series.

In the genus Spilogona, species limits are occasionally ambiguous and species identification based on morphological characters is difficult. In such cases, a molecular-genetic analysis can be applied. Species 
identification and species delimitation are more accurate when based not only on morphological but also on molecular-genetic characters. Unfortunately, there are very few papers devoted to the DNA barcoding of the Muscidae and most of them have used the sequence data to perform phylogenetic analyses (Savage et al. 2004; Schuehli et al. 2004, 2007; Kutty et al. 2008, 2014; Haseyama et al. 2015). Nevertheless, there is one paper in which the relevance of DNA barcoding as a taxonomic tool in muscid fly identification has been established (Renaud et al. 2012).

The aim of this paper is to analyse genetic differences in the cytochrome oxidase I gene of flies of the 'Spilogona contractifrons species-group' and the 'Spilogona nitidicauda species-group', to confirm the morphological differences.

\section{Material and methods}

The material of Spilogona used in this study, including type material, is deposited in the Siberian Zoological Museum of the Institute of Systematics and Ecology of Animals, Russian Academy of Sciences, Siberian Branch, Novosibirsk (SZMN), the Zoological Museum of the Moscow State University, Moscow (ZMUM), the Zoological Institute, Saint Petersburg (ZISP), and the Canadian National Collection of Insects, Ottawa (CNC).

\section{Morphological analyses}

Specimens were examined using an Altami PSO745-T microscope for external morphological characters. For the dissection of the male and female terminalia, the end of abdomen was removed and boiled in a $10 \% \mathrm{KOH}$ solution for 15-20 s. After dissection and study, the abdomen and terminalia were washed and then stored in microvials of glycerine pinned directly underneath the specimens. Pictures were made with the camera Canon EOS 600D. Illustrations were made in ink and then edited using Adobe Photoshop CS.

\section{Molecular-genetics analyses}

The molecular-genetic analysis was based on the study of the COIgene, the most standardized short genetic marker in an organism's DNA that is used as a taxonomic DNA barcoding method (Ratnasingham \& Hebert 2007). Total DNA was extracted from samples of Spilogona using the DIAtom ${ }^{\mathrm{TM}}$ DNA Prep kit (Isogen, Russia). Polymerase chain reaction (PCR) of the cytochrome c oxidase subunit I (COI) locus was conducted with an Encyclo PCR kit (Evrogen, Russia) using TY-J-1460 (Simon et al. 1994) and COIR (Shaikevich 2007) primers. The following amplification conditions were used: initial denaturation at $94^{\circ} \mathrm{C}(5 \mathrm{~min}) ; 35$ cycles: denaturing at $94^{\circ} \mathrm{C}(30 \mathrm{~s})$, annealing at $55^{\circ} \mathrm{C}(30 \mathrm{~s})$, elongation at $72^{\circ} \mathrm{C}(40$ s); and final elongation at $72^{\circ} \mathrm{C}(10 \mathrm{~min})$. The PCR fragments were purified from agarose gel with a Clean-Up Extraction Kit (Evrogen, Russia) and were sequenced using the BigDye Termination kit 3.1 (Applied Biosystems, USA). All sequences were deposited in the GenBank under accessions numbers MH413049-MH413064.

Specimens for genetic analysis were taken from different populations in the territories of Russia. The institution code, the voucher and the GenBank accessions number of specimens are indicated within brackets. A total of 16 specimens of 6 species of Spilogona was examined:

\section{Spilogona alticola}

RUSSIA: 1 ', Altai Republic, Kosh-Agach district, Ukok plateau, environs of Kal'dzhin-Kul'-Bas lake, $49^{\circ} 19^{\prime} \mathrm{N}, 8^{\circ} 26^{\prime}$ E, 2400-2600 m a.s.1., 20 Jul. 2006, V. Sorokina leg. (SZMN SA13 MH413052); 1 ${ }^{\top}$, Altai Republic, Kosh-Agach district, Ukok plateau, environs of Kal'dzhin-Kul'-Bas lake, $49^{\circ} 19^{\prime} \mathrm{N}$, 87²6' E, 2400-2600 m a.s.1., 20 Jul. 2006, V. Sorokina leg. (SZMN SA14 MH413053). 


\section{Spilogona arctica}

RUSSIA: $1 \hat{\jmath}$, Altai Republic, Kosh-Agach district, Severo-Chuyskyi Range, valley of Aktru River, 5006' N, 8748' E, 2064 m a.s.1., 21 Jul. 2013, V. Sorokina leg. (SZMN SA9 MH413051); 1 ○, Krasnoyarsk Kray, Taimyr Peninsula, $12.5 \mathrm{~km}$ to south from Dixon, the mouth of the river Lemberova,

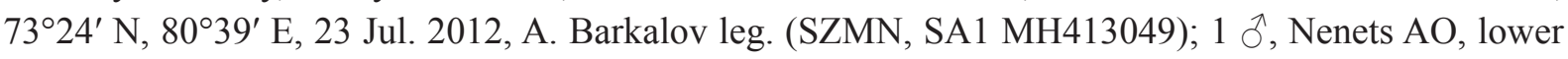

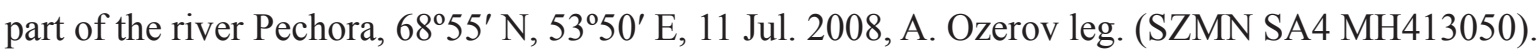

\section{Spilogona contractifrons}

RUSSIA: 1 त, Altai Republic, Kosh-Agach district, Ukok plateau, Rodonovyi spring, upper part of Zhumaly river, $49^{\circ} 27^{\prime} \mathrm{N}, 88^{\circ} 03^{\prime} \mathrm{E}, 2410 \mathrm{~m}$ a.s.1., 4 Jul. 2005, T. Novgorodova leg. (SZMN SC22

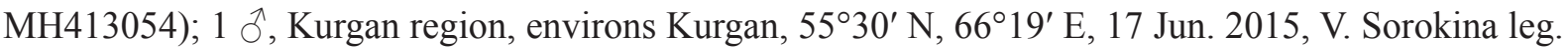

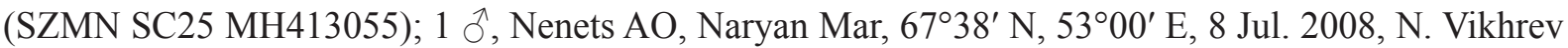

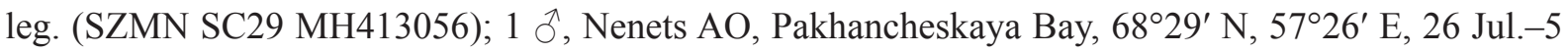
Aug. 2015, O. Makarova and M. Bizin leg. (SZMN SC30 MH413057).

\section{Spilogona imitatrix}

RUSSIA: $1 \hat{\jmath}$, Altai Republic, Ongudai district, Terektinskiy mountain range, upper part of Bolshoi

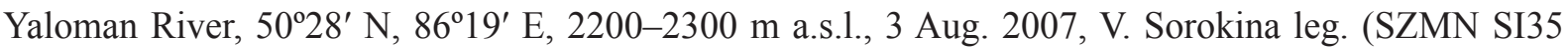
MH413061).

\section{Spilogona nitidicauda}

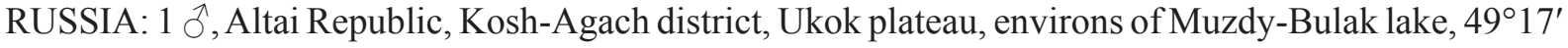
N, 87³9' E, 2450 m a.s.1., 2 Jul. 2005, V. Sorokina leg. (SZMN SN38 MH413062); 1 §, Chukotka AO, Beringovskii district, 40 km SSW Beringovskii, 62 $43^{\prime}$ N, 178 55' E, 22 Jul. 2012, A. Stekolshchikov leg. (SZMN SN40 MH413063); 1 §, Chukotka AO, Beringovskii district, 40 km SSW Beringovskii,

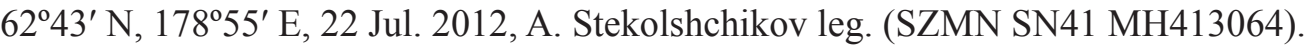

\section{Spilogona platyfrons}

RUSSIA: $1 \partial^{\top}$, Altai Republic, Ulagan district, Shapshal'skiy mountain range, upper part of Shui River, $\sim 50^{\circ} 33^{\prime} \mathrm{N}, 89^{\circ} 50^{\prime} \mathrm{E}, 2550-2900 \mathrm{~m}$ a.s.1., 24 Jul. 2007, V. Sorokina leg. (SZMN SP32 MH413058); 1 ठ, Altai Republic, Ust-Koksa district, 47 km W of Ust-Koksa, Krasnaya Mountains, $50^{\circ} 04^{\prime} \mathrm{N}, 85^{\circ} 13^{\prime} \mathrm{E}$, 2000-2100 m a.s.1., 16 Jul. 2015, V. Sorokina leg. (SZMN SP33 MH413059); 1 O, Altai Republic, Ust-

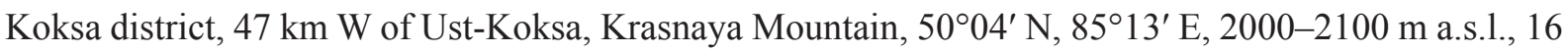
Jul. 2015, V. Sorokina leg. (SZMN SP34 MH413060).

To investigate the genetic relationships of the isolates of Spilogona, Maximum Likelihood phylogenetic trees based on the COI gene nucleotide data were reconstructed; values lower than $50 \%$ were eliminated. A matrix of pairwise distances were estimated using the Maximum Composite Likelihood (MCL) approach. The analysis involved 38 nucleotide sequences and there was a total of 408 positions in the final dataset. Evolutionary analyses were conducted in MEGA6 (Tamura et al. 2013).

The following abbreviations are used in the text: $a=$ anterior; $a c r=$ acrostichal; $a d=$ anterodorsal, $a v=$ anteroventral; $d=$ dorsal; $d c=$ dorsocentral; $p=$ posterior, $p d=$ posterodorsal; $p h=$ posthumeral, post $=$ postsutural; $p s=$ presutural; $p v=$ posteroventral; $v=$ ventral; $\mathrm{AO}=$ Autonomous Okrug; $\mathrm{ML}=$ Maximum Likelihood.

The citation of some original labels is given in quotation marks, but interpretations of these data are given in square brackets. 


\section{Results}

\section{Taxonomic analysis of 'Spilogona contractifrons species-group'}

Flies of this group of species can be distinguished as follows: scutum with 3 post dc setae, mid tibia without $p v$ and with $1 a d$ seta, fore tibia with $1 p$ seta, hind femur with a row of $p v$ setae, mid femur with anterior preapical seta, haltere yellow, facial edge not projecting beyond level of profrons, prementum shining, scutellum without downwardly-directed preapical setulae on upper border of declivities, abdomen plump, katepisternal setae arranged in a more or less equilateral triangle like Coenosia Meigen, 1826.

Four species of this group are currently recognised: the widespread S. alticola, S. arctica, S. contractifrons, and the East Asian S. orthosurstyla. Research for the current paper has included a detailed study of extensive material from the territory of Russia, from both tundra zones and mountain areas, and personal study of the bulk of Huckett's collection deposited in the Canadian National Collection, Ottawa, Canada.

The study of extensive material has confirmed the existence of four species in this group. Despite the difficulties in the recognition of species of this group, there are several helpful diagnostic characters: the shape of male sternite 5, the shape of male terminalia, and the colour of the scutum (Figs 1-2, Table 1). The colour of the scutum can be variable in different populations but the shape of the male sternite 5 is constant and is clearly visible in dry specimens (Fig. 1 G-I).

At present it is impossible to separate females of these species. Males can be distinguished by the following couplets:

1. Scutum completely dark brown (mountain populations) or light grey with indistinct stripes (arctic tundra populations); sternite 5 with a narrow median notch (Fig. 1E, H), with a wavy inner edge; in lateral view hypandrium with a protuberance; surstylus sharply curved at middle (Fig. 1K)

S. arctica (Zetterstedt, 1838)

- Scutum grey or brownish dusted with more or less distinct dark stripes; sternite 5 with a rather wide median notch; hypandrium without a distinct protuberance; shape of surstylus not as above ......... 2

2. Sternite 5 with a wide rectangular median notch, inner edge more or less straight (Fig. 1F, I); scutum with distinct dark stripes; surstylus short, gently curved, about as long as cerci (Fig. 1L) S. alticola (Malloch, 1920)

- Sternite 5 not as above; scutum with indistinct dark stripes, often diffuse; surstylus longer than cerci

3. Sternite 5 with an extended and pointed caudal margin (Fig. 2C); surstylus straight, curved only at apex (Fig. 2A)

S. orthosurstyla Xue \& Tian, 1988

- Sternite 5 with a rounded caudal margin (Fig. 1D, G); surstylus not straight, weakly curved at middle (Fig. 1J)

S. contractifrons (Zetterstedt, 1838) 

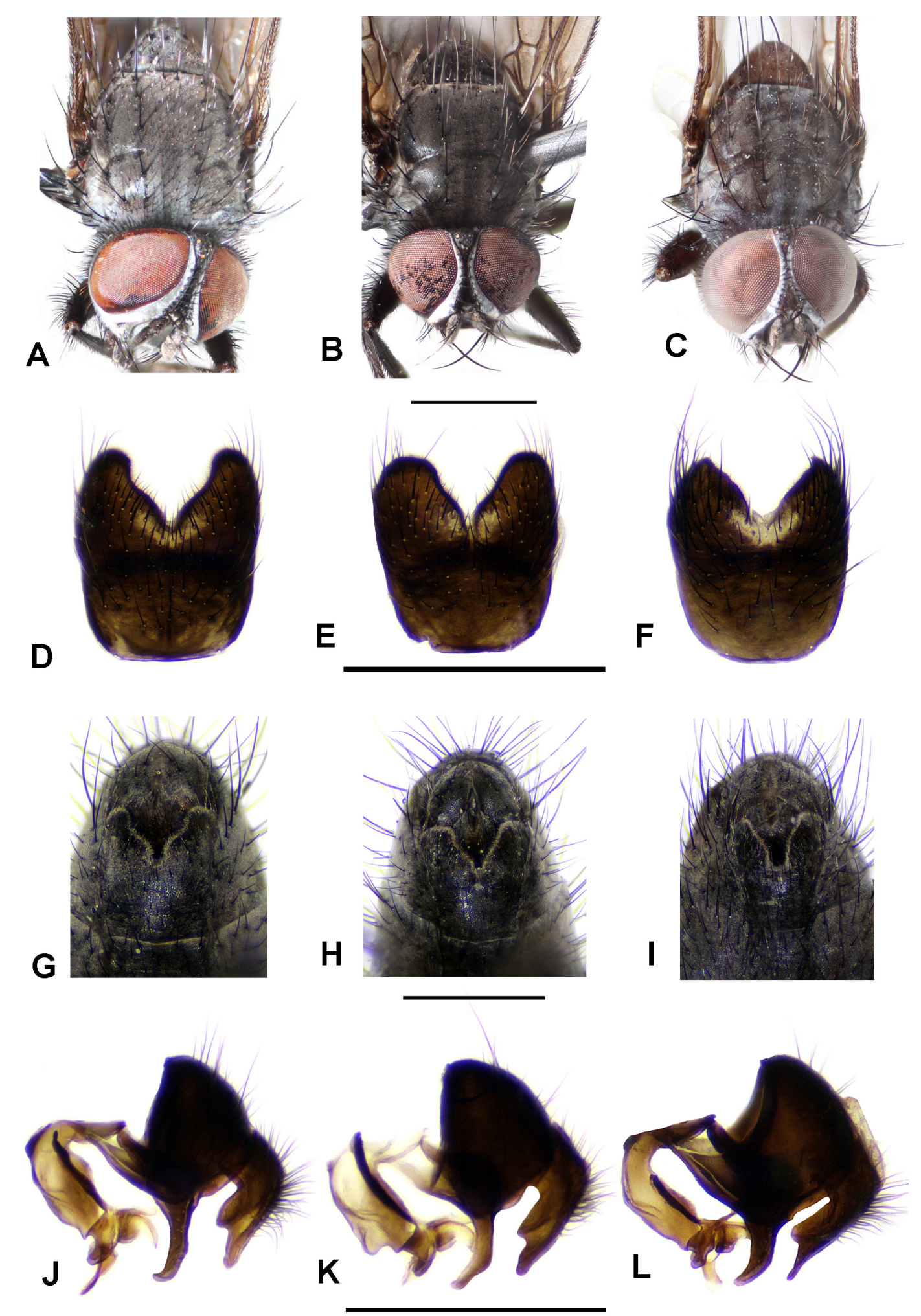

Fig. 1. Males of Spilogona Schnabl, 1911. A-C. Male head and scutum, anterior view. A. S. contractifrons (Zetterstedt, 1838). B. S. arctica (Zetterstedt, 1838). C. S. alticola (Malloch, 1920). D-F. Sternite 5. D. S. contractifrons. E. S. arctica. F. S. alticola. G-I. Abdomen, dorsal view. G. S. contractifrons. H. S. arctica. I. S. alticola. J-L. Terminalia, lateral view. J. S. contractifrons. K. S. arctica. L. S. alticola. Scale bars: $1 \mathrm{~mm}$. 


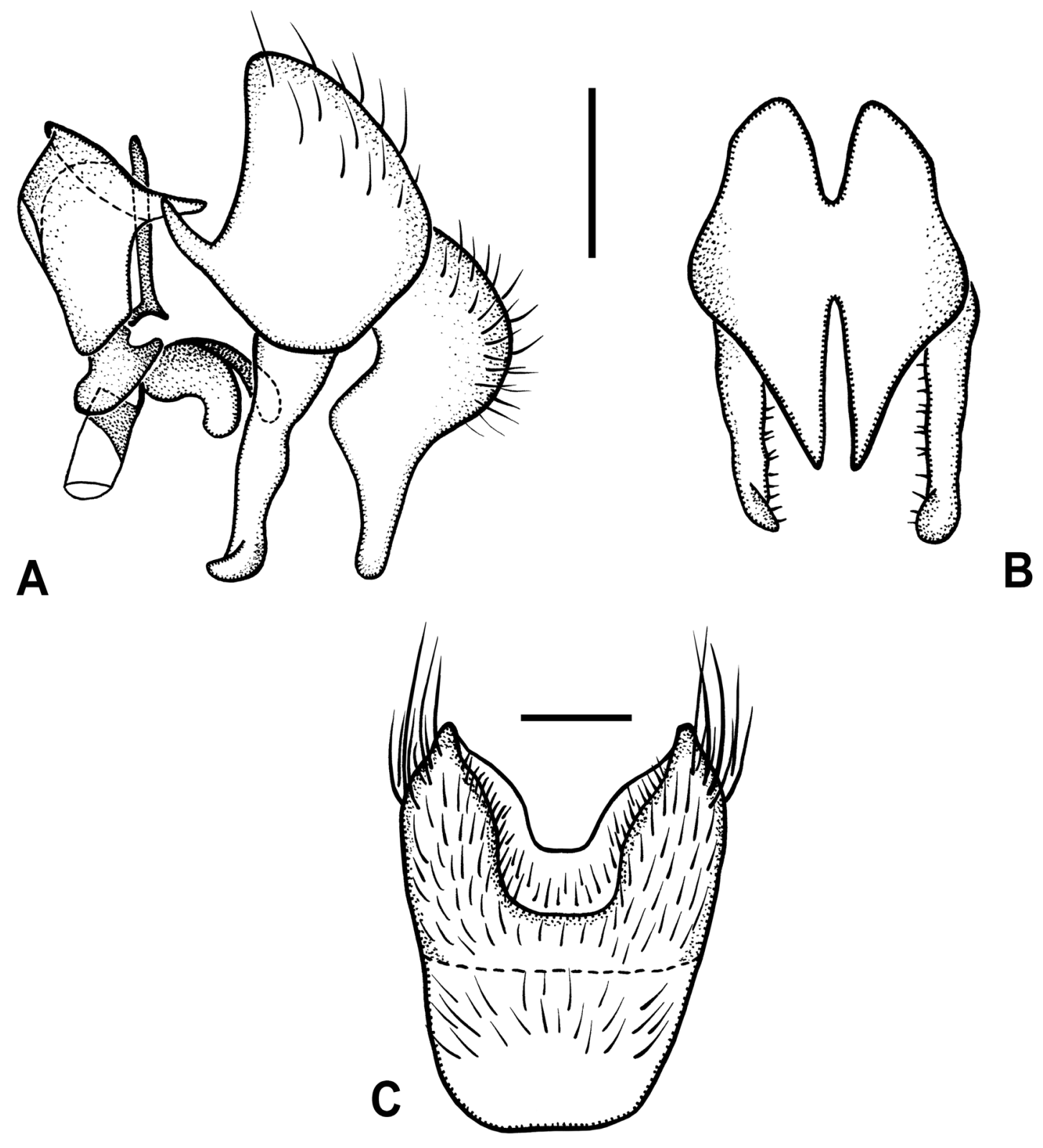

Fig. 2. Spilogona orthosurstyla Xue \& Tian, 1988. A. Terminalia, lateral view. B. Terminalia, dorsal view. C. Sternite 5. Scare bars: $0.25 \mathrm{~mm}$. 
Table 1. Diagnostic characters of males of 'Spilogona contractifrons species-group'.

\begin{tabular}{|c|c|c|c|c|}
\hline & $\begin{array}{l}\text { S. alticola } \\
\text { (Malloch, 1920) }\end{array}$ & $\begin{array}{l}\text { S. arctica } \\
\text { (Zetterstedt, 1838) }\end{array}$ & $\begin{array}{l}\text { S. contractifrons } \\
\text { (Zetterstedt, 1838) }\end{array}$ & $\begin{array}{l}\text { S. orthosurstyla } \\
\text { Xue \& Tian, } 1988\end{array}$ \\
\hline Colour of scutum & $\begin{array}{l}\text { grey and brown } \\
\text { dusted with } \\
\text { distinct dark } \\
\text { stripes (Fig. 1C) }\end{array}$ & $\begin{array}{l}\text { completely dark } \\
\text { brown (mountains) } \\
\text { or grey dusted } \\
\text { (arctic tundra) with } \\
\text { indistinct dark } \\
\text { stripes (Fig. 1B) }\end{array}$ & $\begin{array}{l}\text { grey or brownish-grey } \\
\text { dusted with brown } \\
\text { stripes, sometimes } \\
\text { diffuse (Fig. 1A) }\end{array}$ & $\begin{array}{l}\text { brown dusted with } \\
\text { indistinct dark } \\
\text { stripes }\end{array}$ \\
\hline $\begin{array}{l}\text { Shape of } \\
\text { sternite } 5\end{array}$ & $\begin{array}{l}\text { with a wide } \\
\text { median notch, } \\
\text { inner edge more } \\
\text { or less straight, } \\
\text { with a rounded } \\
\text { caudal margin } \\
\text { (Fig. 1F, 1I) }\end{array}$ & $\begin{array}{l}\text { with a narrow } \\
\text { median notch, inner } \\
\text { edge wavy, with } \\
\text { a rounded caudal } \\
\text { margin (Fig. 1E, } \\
1 \mathrm{H})\end{array}$ & $\begin{array}{l}\text { with a fairly wide } \\
\text { median notch, the inner } \\
\text { edge strongly curved, } \\
\text { with a rounded caudal } \\
\text { margin (Fig. 1D, 1G) }\end{array}$ & $\begin{array}{l}\text { with a fairly wide } \\
\text { median notch, } \\
\text { the inner edge } \\
\text { not curved, with } \\
\text { extended and } \\
\text { pointed caudal } \\
\text { margin (Fig. 2C) }\end{array}$ \\
\hline $\begin{array}{l}\text { Shape of } \\
\text { surstylus }\end{array}$ & $\begin{array}{l}\text { short, gently } \\
\text { curved (Fig. 1L) }\end{array}$ & $\begin{array}{l}\text { sharply curved at } \\
\text { middle (Fig. 1K) }\end{array}$ & $\begin{array}{l}\text { weakly curved at } \\
\text { middle (Fig. 1J) }\end{array}$ & $\begin{array}{l}\text { straight, curved } \\
\text { only at apex } \\
\text { (Fig. 2A) }\end{array}$ \\
\hline $\begin{array}{l}\text { Shape of } \\
\text { hypandrium }\end{array}$ & $\begin{array}{l}\text { without processes, } \\
\text { or with a small } \\
\text { tubercle, narrow }\end{array}$ & $\begin{array}{l}\text { with protuberance } \\
\text { (Fig. } 1 \mathrm{~K} \text { ) }\end{array}$ & without protuberance & $\begin{array}{l}\text { without } \\
\text { protuberance }\end{array}$ \\
\hline
\end{tabular}

Family Muscidae Latreille, 1802

Subfamily Coenosiinae Verrall, 1888

Tribe Limnophorini Villeneuve, 1902

Genus Spilogona Schnabl, 1911

Spilogona alticola (Malloch, 1920)

Fig. 1C, F, I, L

\section{Type locality}

USA: New Hampshire.

\section{Material examined}

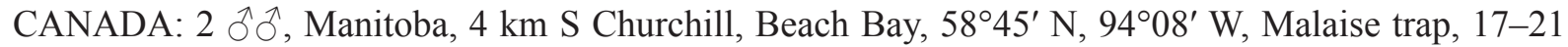
Jul. 2007, A. Renaud leg. (SZMN); 1 + , same data as for preceding except: pan traps, 25-29 Jul. 2007

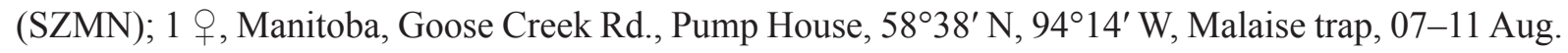
2007, A. Renaud leg. (SZMN).

RUSSIA: 14 え๐, Altai Republic, Kosh-Agach district, Ukok plateau, environs of Muzdy-Bulak lake, $49^{\circ} 17^{\prime} \mathrm{N}, 87^{\circ} 39^{\prime} \mathrm{E}, 2400-2900 \mathrm{~m}$ a.s.1., sweep in vegetation, 26 Jun. 2005, 1 Jul. 2005, A. Barkalov and V. Sorokina leg. (SZMN); $2 \widehat{\partial}$, Altai Republic, Kosh-Agach district, Ukok plateau, Rodonovyi spring, upper part of Zhumaly river, $49^{\circ} 27^{\prime} \mathrm{N}, 88^{\circ} 03^{\prime} \mathrm{E}, 2410 \mathrm{~m}$ a.s.l., sweep in vegetation, 24 Jun. 2005, V. Sorokina leg. (SZMN); $13 \hat{\partial} \widehat{\partial}$, Altai Republic, Kosh-Agach district, Ukok plateau, environs of 


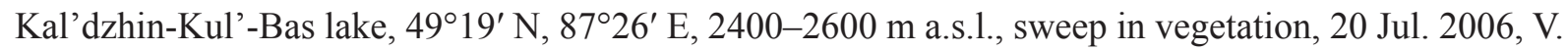
Sorokina and T. Novgorodova leg. (SZMN); $1 \overbrace{}^{\top}$, Chukotka AO, lower part of the Anadyr river, $64^{\circ} 50^{\prime}$ N, 17558' E, 5 m a.s.1., 20 Jul. 2013, A. Barkalov leg. (SZMN).

\section{Distribution}

Holarctic Region; in the Palaearctic, from Southern Siberia to the north of the Russian Far East. Arctoalpine species.

Spilogona arctica (Zetterstedt, 1838)

Fig. 1B, E, H, K

\section{Type locality}

GREENLAND.

\section{Material examined}

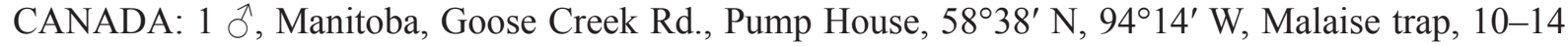
Jul. 2007, A. Renaud leg. (SZMN).

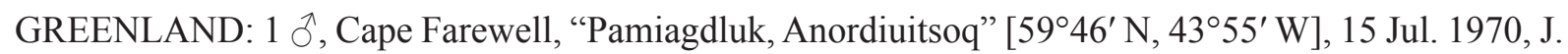
Böcher leg. (SZMN); 1 q, same data as for preceding except: 1 Aug. 1970 (SZMN).

RUSSIA: $6 \hat{\partial}$, Altai Republic, Turochak district, $8 \mathrm{~km} \mathrm{~S}$ of Obogo village, south slope of Archa Mountain, [ $\sim 51^{\circ} 26^{\prime} \mathrm{N}, 87^{\circ} 19^{\prime}$ E], 1250 m a.s.1., 25 Jun. 2003, V. Sorokina leg. (SZMN); 2 ठ઼ 9 우우, Altai Republic, Shebalino district, environs of Mukhorcherga village, Belog-Mukhorcherga Mountain, $51^{\circ} 20^{\prime} \mathrm{N}, 85^{\circ} 19^{\prime} \mathrm{E}, 1548-1854 \mathrm{~m}$ a.s.1., 22 Jul. 2011, V. Sorokina leg. (SZMN), 1 đ’, Altai Republic, Seminsky Pass, $51^{\circ} 03^{\prime} \mathrm{N}, 85^{\circ} 35^{\prime} \mathrm{E}, 1650 \mathrm{~m}$ a.s.1., 27-30 Jun. 2016, N. Vikhrev leg. (ZMUM); 2 ○ึ

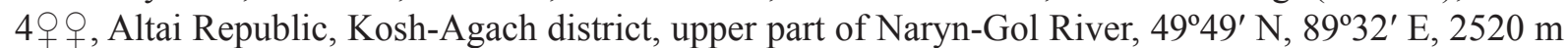
a.s.1., 15-19 Jul. 2009, V. Sorokina and T. Novgorodova leg. (SZMN); 5 đิ 0 5 우, Altai Republic, Kosh-Agach district, south slope of Yuzhno-Chuiskiy range, valley of Tara River, $49^{\circ} 39^{\prime} \mathrm{N}, 88^{\circ} 13^{\prime} \mathrm{E}$,

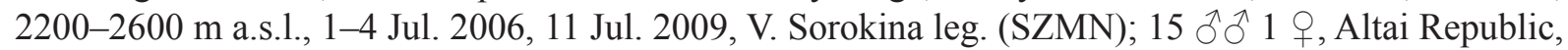
Kosh-Agach district, Ukok plateau, Rodonovyi spring, upper part of Zhumaly river, $49^{\circ} 27^{\prime} \mathrm{N}, 88^{\circ} 03^{\prime} \mathrm{E}$, 2410 m a.s.1., 24 Jun. 2005, 4 Jul. 2005, 23-24 Jul. 2006, V. Sorokina, A. Barkalov and T. Novgorodova leg. (SZMN); 32 ㅎำ 7 우우, Altai Republic, Kosh-Agach district, 6-8 km NW Maitobe Mountain, $49^{\circ} 34^{\prime}$ N, $87^{\circ} 43^{\prime}$ E, 2400-2800 m a.s.1., 6-9 Jul. 2006, V. Sorokina and T. Novgorodova leg. (SZMN);

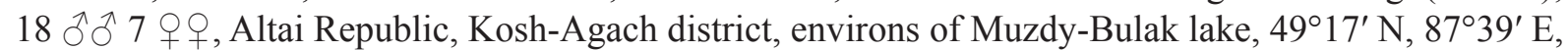

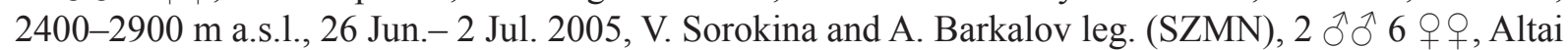
Republic, Kosh-Agach district, environs of Kal'dzhin-Kul'-Bas lake, $49^{\circ} 19^{\prime}$ N, 87²6' E, 2400-2600 m

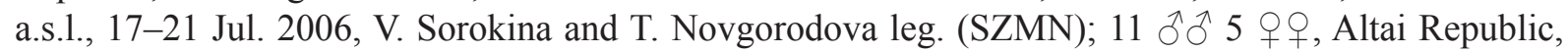

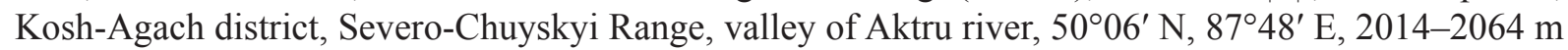
a.s.l., 21 Jul. 2013, V. Sorokina and T. Novgorodova leg. (SZMN); 1 + , Altai Republic, Kosh-Agach district, south slope of Kurayskyi mountain ridge, 50 $0^{\circ} 8^{\prime} \mathrm{N}, 87^{\circ} 51^{\prime} \mathrm{E}, 2736 \mathrm{~m}$ a.s.l., 19 Jul. 2013, V. Sorokina leg. (SZMN); $1 \partial^{\circ}$, Altai Republic, Ulagan district, upper part of Ioldu river, $50^{\circ} 50^{\prime} \mathrm{N}, 88^{\circ} 57^{\prime}$ E, 2000 m a.s.1., 18 Jun. 2005, V. Sorokina leg. (SZMN); 10 ํำ 3 우오, Altai Republic, Ulagan district,

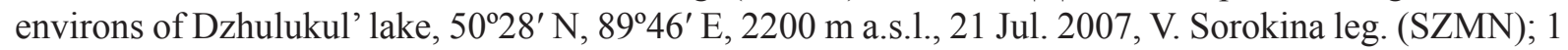
+ , Altai Republic, Ongudai district, Terektinskiy range, upper part of Bolshoi Yaloman river, $50^{\circ} 28^{\prime} \mathrm{N}$,

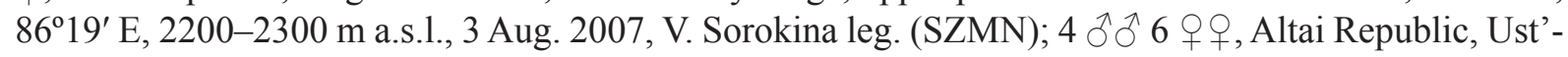
Koksa district, Nizhne-Multinskoe lake, 5001' N, 8549' E, 1633 m a.s.l., 13 Jul. 2013, V. Sorokina leg.

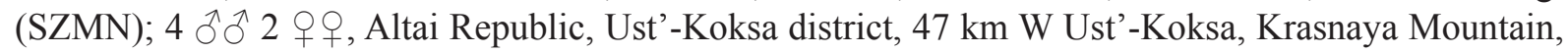

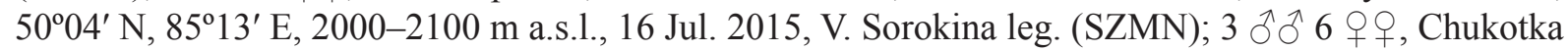

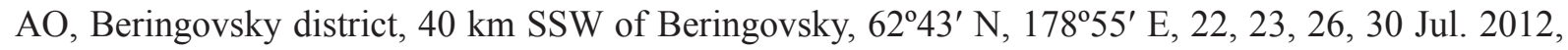




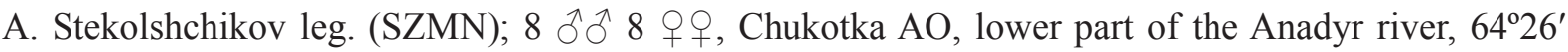

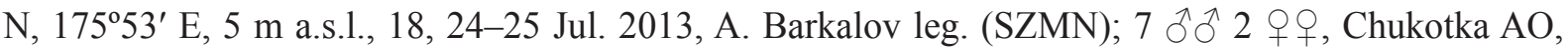
30 km NW Krasneno, Anadyr river, 64 ${ }^{\circ} 46^{\prime}$ N, 174 ${ }^{\circ} 8^{\prime}$ E, 6-19 Jul. 2014, A. Barkalov and V. Zinchenko

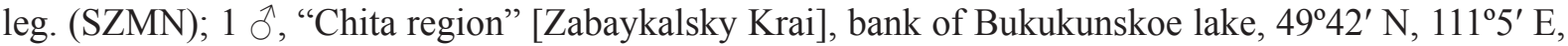
1880 m a.s.1., 3 Aug. 1991, V. Pekin leg. (SZMN); 1 §ै, "Chita region" [Zabaykalsky Krai], Sokhondo Nature Reserve, 49 $41^{\prime}$ N, $111^{\circ} 5^{\prime}$ E, 1350-1400 m a.s.l., 2 Jun. 1991, V. Pekin and D. Logunov leg. (SZMN); 7 Oे, Krasnoyarsk Kray, environs of Norilsk, Talnakh, $69^{\circ} 21^{\prime} \mathrm{N}, 87^{\circ} 45^{\prime} \mathrm{E}$, birch forest, 10 ,

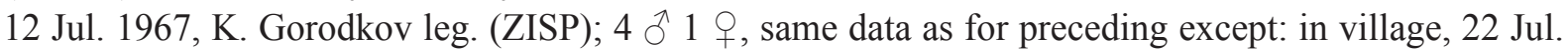
1973; 1 J, Krasnoyarsk Kray, upper part of Pura river [left tributary of Pyasina river], $10 \mathrm{~km}$ NW of Berasmu lake [ $71^{\circ} 51^{\prime} \mathrm{N}, 87^{\circ} 30^{\prime}$ E], willow in bank of river, 21 Jul. 1967, K. Gorodkov leg. (ZISP); $6 \bigcirc^{\lambda} 1$ + , Krasnoyarsk Kray, Taymyr Peninsula, $90 \mathrm{~km} \mathrm{~S}$ of Tareya village, Pyasina river, [ $72^{\circ} 25^{\prime} \mathrm{N}, 90^{\circ} 56^{\prime} \mathrm{E}$ ], willow in bank of river, 23 Jul. 1967, K. Gorodkov leg. (ZISP); 4 ô $\sigma^{\lambda}$, , Krasnoyarsk Kray, Taymyr

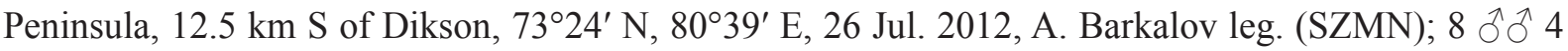
ㅇ, Krasnoyarsk Kray, Taymyr Peninsula, $20 \mathrm{~km}$ NW of Khatanga, Ary-Mas station, $72^{\circ} 03^{\prime} \mathrm{N}, 101^{\circ} 56^{\prime}$ E, 14 m a.s.1., 10-22 Jul. 2010, A. Barkalov leg. (SZMN); 2 우, Krasnoyarsk Kray, Taymyr Peninsula, $114 \mathrm{~km} \mathrm{~S}$ of Khatanga, Kotuy river, $71^{\circ} 02^{\prime} \mathrm{N}, 103^{\circ} 00^{\prime}$ E, 2-5 Jul. 2010, A. Barkalov leg. (SZMN); 2

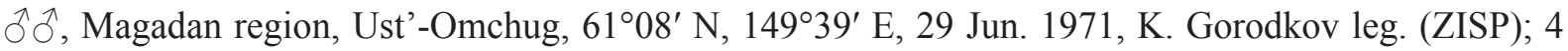
$\lambda \partial^{\lambda}$, same data as for preceding except: larch woodlands, 1 Jul. 1971; 2 우, same data as for preceding except: bog, 1 Aug. 1971, K. Gorodkov and Chelnokov leg. (ZISP); 1 ô, Magadan region, $20 \mathrm{~km} \mathrm{~S}$ of Gizhiga village, "Chaibukha aerodrome", [ 61 ${ }^{\circ} 50^{\prime}$ N, $160^{\circ} 32^{\prime}$ E], 2 Sep. 1987, K. Gorodkov leg.

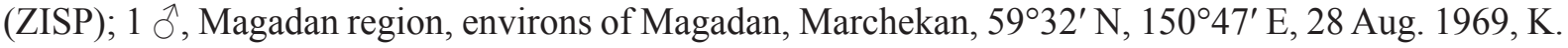
Gorodkov leg. (ZISP); 1 1 ㅇ, Magadan region, the motorway R-504, Yablonevyi Pass, $120 \mathrm{~km}$ NNE of Magadan, $60^{\circ} 35^{\prime} \mathrm{N}, 151^{\circ} 32^{\prime}$ E, $900 \mathrm{~m}$ a.s.1., 17 Jul. 2014, N. Vikhrev leg. (ZMUM); 6 ठ $^{\lambda}$, Nenets AO, lower part of the Pechora river, $68^{\circ} 20^{\prime}$ N, $53^{\circ} 18^{\prime}$ E, 11 Jul. 2008, A. Ozerov leg. ( $2 \delta^{\lambda} \mathrm{SZMN}, 4$

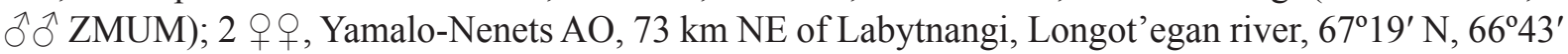
E, 175 m a.s.1., 6, 22 Jul. 2015, A. Barkalov and V. Zinchenko leg. (SZMN).

SWEDEN: 3 $ぇ ึ 1$ ๆ, Abisko, Nuolia Mountain, Ridonjira, heath, 29 Jul. 1995, A. Pont leg. (SZMN).

USA: 1 \ 1 , Alaska, Aleutian Islands, Chowiet Island, 56 $02^{\prime} \mathrm{N}, 156^{\circ} 44^{\prime} \mathrm{W}, 107 \mathrm{~m}$ a.s.l., sweep in grass meadow, 10 Jul. 2009, A. Renaud leg. (SZMN).

\section{Distribution}

Holarctic Region; in the Palaearctic, from Greenland and Northern Europe to the Russian Far East. Arcto-alpine species.

Spilogona contractifrons (Zetterstedt, 1838)

Fig. 1A, D, G, J

\section{Type locality}

SWEDEN: "Lapland".

\section{Material examined}

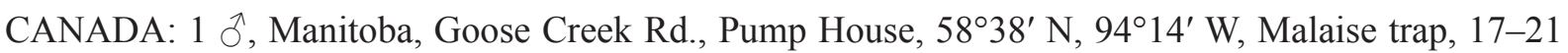
Jul. 2007, A. Renaud leg. (SZMN); 1 Oे, Manitoba, $4 \mathrm{~km} \mathrm{~S}$ of Churchill, Beach Bay, $58^{\circ} 45^{\prime} \mathrm{N}, 94^{\circ} 08^{\prime}$ W, sweep in vegetation, 24 Jul. 2007, A. Renaud leg. (SZMN); 1 q, Manitoba, Churchill, $12 \mathrm{~km} \mathrm{E} \mathrm{on}$ Launch road, 58 $45^{\prime} \mathrm{N}, 93^{\circ} 59^{\prime} \mathrm{W}$, Malaise trap, 3-7 Jul. 2007, A. Renaud leg. (SZMN); 1 + , Manitoba,

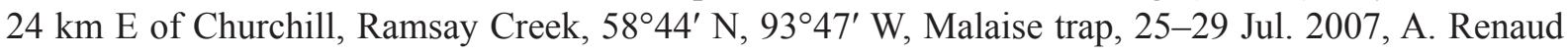
leg. (SZMN). 
RUSSIA: 1 ${ }^{\lambda}$, Altai Republic, Kosh-Agach district, Ukok plateau, environs of Kal'dzhin-Kul'-Bas lake, $49^{\circ} 19^{\prime} \mathrm{N}, 87^{\circ} 26^{\prime}$ E, $2450 \mathrm{~m}$ a.s.1., 21 Jul. 2006, V. Sorokina leg. (SZMN); $2 \jmath^{\lambda}$, Altai Republic, KoshAgach district, Rodonovyi spring, upper part of Zhumaly river, $49^{\circ} 27^{\prime} \mathrm{N}, 88^{\circ} 03^{\prime} \mathrm{E}, 2410 \mathrm{~m}$ a.s.l., 4 Jul. 2005, 23-24 Jul. 2006, T. Novgorodova and V. Sorokina leg. (SZMN); 1 त, Altai Republic, KoshAgach district, Severo-Chuyskyi Ridge, valley of Aktru River, $50^{\circ} 06^{\prime} \mathrm{N}, 87^{\circ} 48^{\prime} \mathrm{E}, 2064 \mathrm{~m}$ a.s.l., 21 Jul. 2013, V. Sorokina leg. (SZMN); 2 우, "Chita region" [Zabaykalsky Krai], 6 km N, of Teli Mountain,

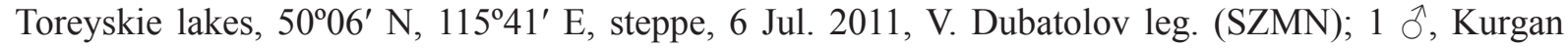

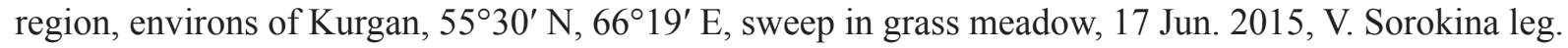

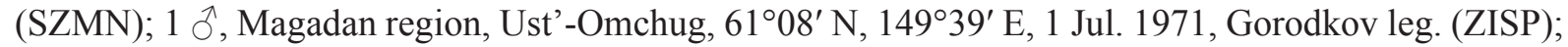
1 1 on, Magadan region, $20 \mathrm{~km} \mathrm{~S}$ of Gizhiga village, "Chaibukha aerodrome" [ $\sim 61^{\circ} 50^{\prime} \mathrm{N}, 160^{\circ} 32^{\prime}$ E], valley of Chaibukha River, $7 \mathrm{~km}$ from mouth of river, 2 Sep. 1987, Gorodkov leg. (ZISP); 1 q, Magadan region, Aborigen field station, $61^{\circ} 20^{\prime} \mathrm{N}, 149^{\circ} 23^{\prime}$ E, $500 \mathrm{~m}$ a.s.1., 5-10 Aug.1990, M. Wood

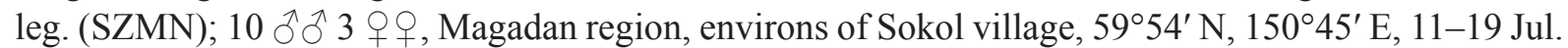
2014, N. Vikhrev leg. (1 91 ô in SZMN, rest in ZMUM); 1 ô, Magadan region, $92 \mathrm{~km}$ NE of Magadan, valley of Donyshko river, $60^{\circ} 20^{\prime} \mathrm{N}, 151^{\circ} 23^{\prime} \mathrm{E}, 570 \mathrm{~m}$ a.s.1., 17 Jul. 2014, N. Vikhrev leg. (ZMUM); 1 ○, Magadan region, Koni Peninsula, environs of Cape Ploskyi cordon, valley of Khindzha river, $59^{\circ} 09^{\prime} \mathrm{N}$, $151^{\circ} 38^{\prime}$ E, 29 Jun. 2016, N. Tridrikh leg. (SZMN); 2 + + , same data as for preceding except: birch copse on the slope, 22 Aug. 2017; 1 , Magadan region, Koni Peninsula, Cape Skalistyi, valley of Skalistaya river, $59^{\circ} 07^{\prime} \mathrm{N}, 151^{\circ} 23^{\prime}$ E, yellow plate traps, 23 Aug. 2017, N. Tridrikh leg. (SZMN); 1 ` 2 우,

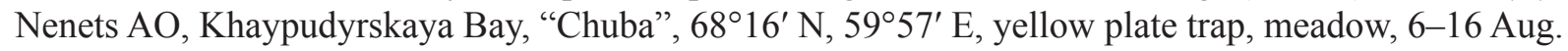

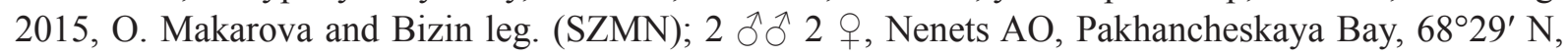
$57^{\circ} 26^{\prime}$ E, yellow plate trap, tundra, 26 Jul.-5 Aug. 2015, O. Makarova and Bizin leg. (SZMN); 1 § 1 , , Nenets AO, Bolvanskaya Bay, $68^{\circ} 05^{\prime} \mathrm{N}, 54^{\circ} 47^{\prime}$ E, yellow plate trap, anthropogenic meadow, 18-25 Jul. 2015, O. Makarova and Bizin leg. (SZMN); 1 \ 4 q $ᄋ$, Novosibirsk region, Zdvinsk district, environs of Shirokaya Kurya village [ $54^{\circ} 34^{\prime}$ N, $78^{\circ} 08^{\prime}$ E], field station, 8 Jul. 2002, Zavarukhina leg. (SZMN).

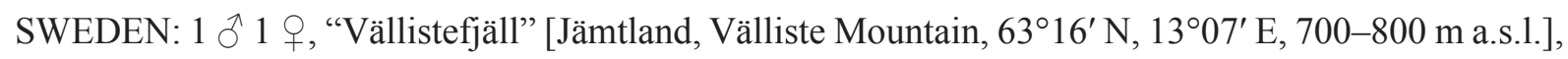
forest zone, 23 Jul. 1980, A. Pont leg. (SZMN).

\section{Distribution}

Holarctic Region; in the Palaearctic, from Northern Europe to the Russian Far East. Arcto-borealmountain species.

Spilogona orthosurstyla Xue \& Tian, 1988

Fig. 2A-C

\section{Type locality}

CHINA: Hebei.

\section{Material examined}

RUSSIA: $1{ }^{\top}$, Altai Republic, Turochak district, $30 \mathrm{~km} \mathrm{SW}$ of Iogach, $51^{\circ} 31^{\prime} \mathrm{N}, 87^{\circ} 23^{\prime} \mathrm{E}, 1600 \mathrm{~m}$ a.s.1., 21 Jun. 2003, V. Sorokina leg. (SZMN).

\section{Distribution}

East Palaearctic: China (Hebei, Shanxi), Russia (Altai Mts). East Asian mountain species.

\section{Remark}

In view of the distribution of other species of this group, we can assume that this species also lives in the arctic zone. 
Table 2. Estimates of evolutionary divergence between studied species of Spilogona Schnabl, 1911. The number of base substitutions per site from averaging over all sequence pairs between groups are shown. The analysis involved 16 nucleotide sequences.

\begin{tabular}{llrrrrr}
\hline & 1 & 2 & 3 & 4 & 5 \\
\hline 1 & S. alticola (Malloch, 1920) & & & & & \\
2 & S. contractifrons (Zetterstedt, 1838) & 0.006 & & & & \\
3 & S. arctica (Zetterstedt, 1838) & 0.018 & 0.018 & & & \\
4 & S. imitatrix (Malloch, 1921) & 0.046 & 0.039 & 0.039 & & \\
5 & S. nitidicauda (Schnabl, 1911) & 0.057 & 0.051 & 0.053 & 0.016 & \\
6 & S. platyfrons Sorokina, 2018 & 0.055 & 0.049 & 0.049 & 0.012 & 0.004 \\
\hline
\end{tabular}

\section{Molecular-genetic analysis of 'Spilogona contractifrons species-group'}

Since in our collection there is only one specimen of $S$. orthosurstyla and it is not in good condition, the molecular analysis was performed only on the other three species of this group.

Genetic distances between species are shown in the ML tree (Fig. 3) and in Table 2. Regardless of the geographic origin of the specimens, all three studied species in this group have identical COI gene sequences within the species. At the same time, at least five nucleotide substitutions (single nucleotide polymorphisms (SNPs)) vary between $S$. alticola and S. contractifrons. Spilogona arctica differs from S. alticola with 13 SNP and from $S$. contractifrons with 12 SNP; one substitution results in amino acid change (M112L).

The genetic distance between $S$. alticola and $S$. contractifrons is $0.6 \%$, and between each of these species and $S$. arctica is $1.8 \%$. All three species formed a distinct cluster on the ML tree with high (98-99\%) statistical support in which the associated taxa clustered together (Fig. 3).

The following results were obtained after comparing the sequences of the studied specimens with the annotated sequences in Genbank. Spilogona arctica from the Altai, Taimyr and from the Nenets AO is identical with $S$. arctica from Sweden. All of them are distinguished by a single substitution of C438T from $S$. arctica from Canada and Greenland (Supplementary file). Spilogona contractifrons from the Altai, Nenets AO, Kurgan and Naryan-Mar is identical in the COI gene with S. contractifrons from Norway and Sweden. But S. alticola from the Altai clustered with S. contractifrons from Canada (Fig. 3).

\section{Taxonomic analysis of 'Spilogona nitidicauda species-group'}

Flies of this group of species can be distinguished as follows: scutum with 4 post $d c$ setae, anepisternum with interspatial setae, facial edge not projecting beyond level of profrons, hind femur without $p v$ setae, scutellum without downwardly-directed preapical setulae on upper border of declivities, abdomen flattened with large trapezoidal marks on tergite 3.

Four species of this group are currently recognised: S. nitidicauda, S. hissarensis, S. platyfrons and S. imitatrix. Spilogona hissarensis was studied only from the original type-series. The other species of this group were studied with material from the territory of Russia and from Huckett's collection deposited in the Canadian National Collection, Ottawa, Canada.

Despite the difficulty in distinguishing the species of this group, there are several helpful diagnostic characters: the width of frons and parafacial, presence of pollen on the prementum, the colour of the scutum and abdomen, the shape of male terminalia (Figs 4-5). Additional diagnostic characters are given in Table 3. 


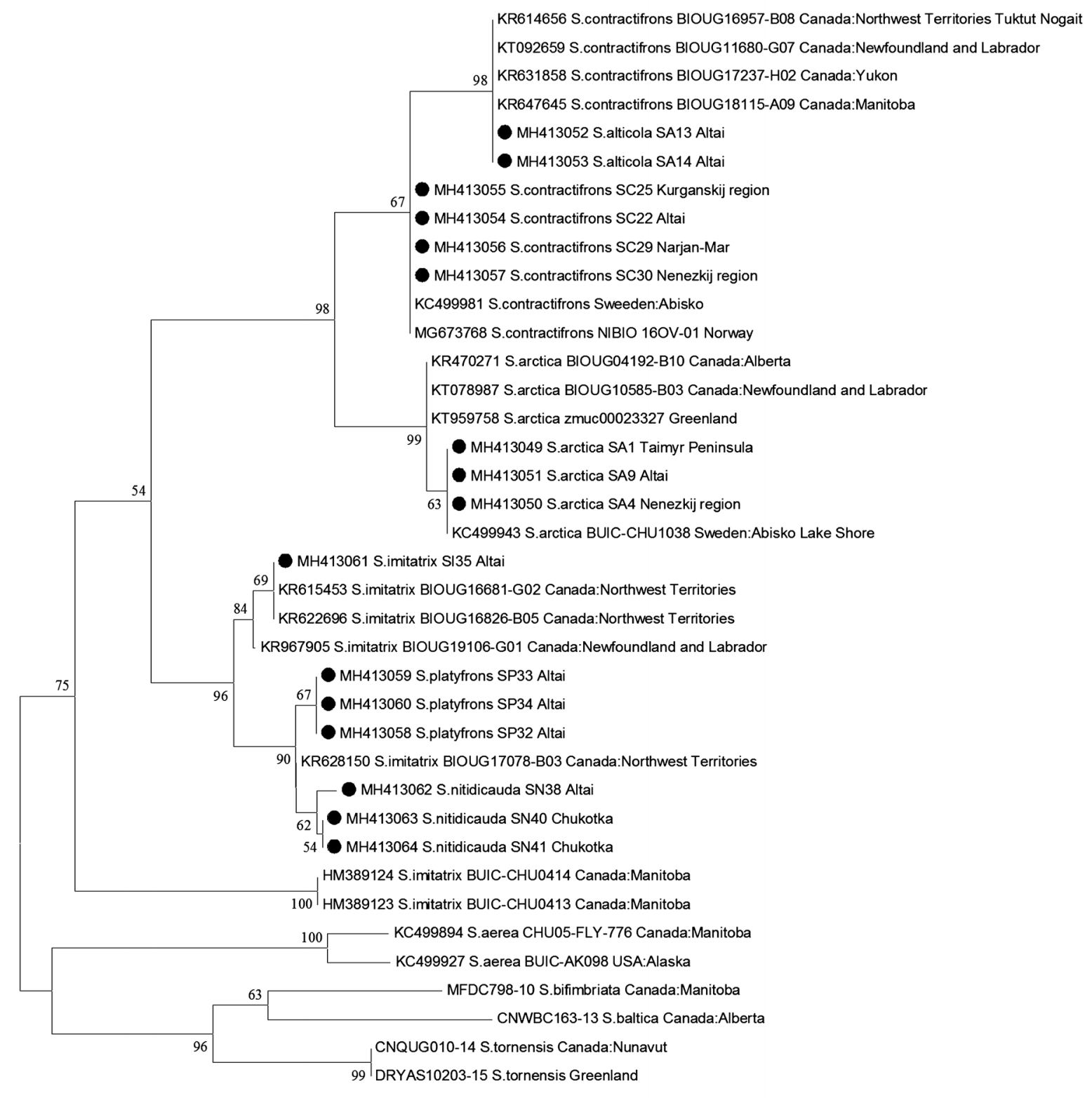

Fig. 3. Maximum Likelihood phylogenetic tree shows evolutionary relationships of species of Spilogona Schnabl, 1911. 

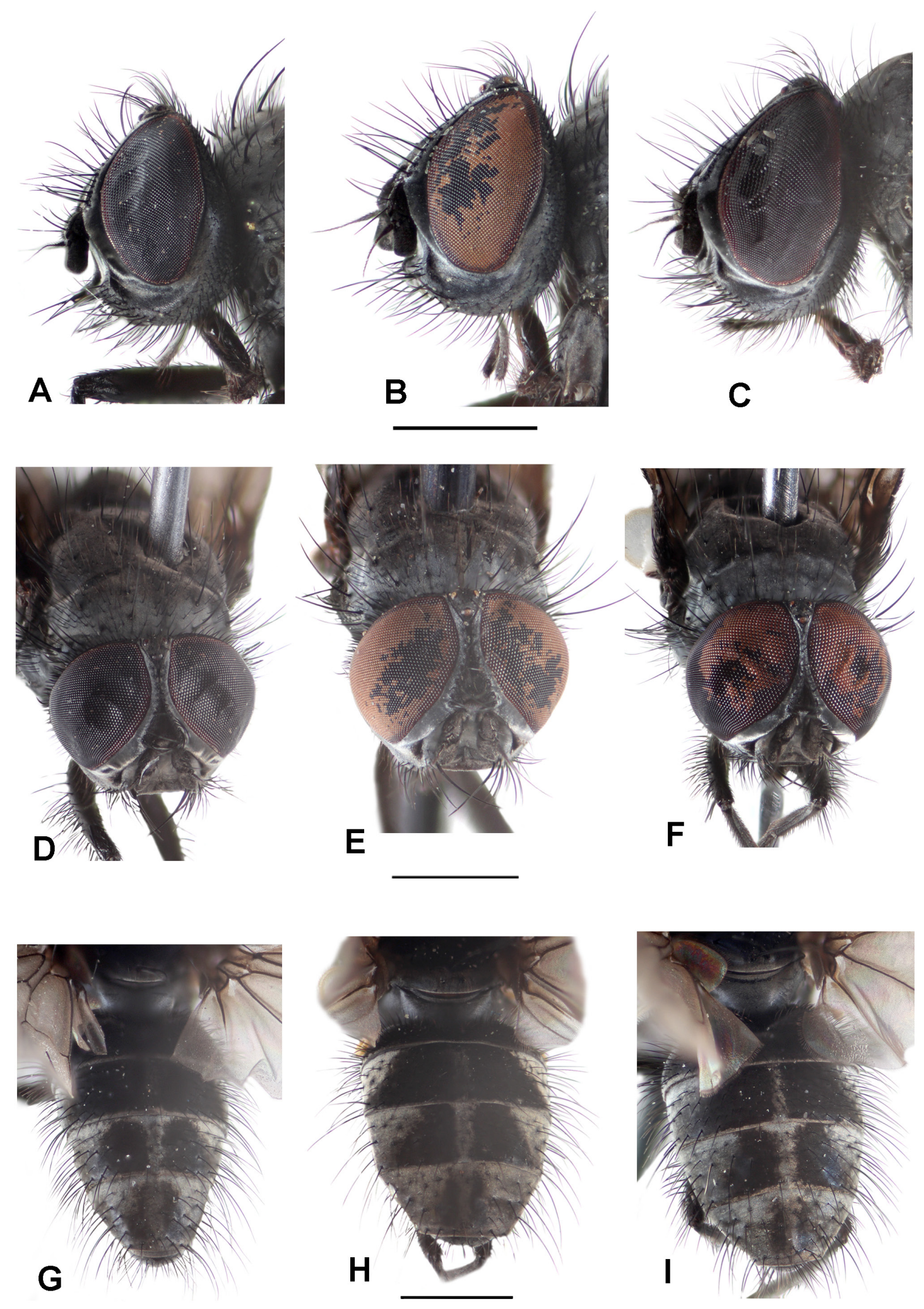

Fig. 4. Males of Spilogona Schnabl, 1911. A-C. Head, lateral view. A. S. imitatrix (Malloch, 1921). B. S. nitidicauda (Schnabl, 1911). C. S. platyfrons Sorokina, 2018. D-F. Frons, anterior view. D. S. imitatrix. E. S. nitidicauda. F. S. platyfrons. G-I. Abdomen, dorsal view. G. S. imitatrix. H. S. nitidicauda. I. S. platyfrons. Scale bars: $1 \mathrm{~mm}$. 

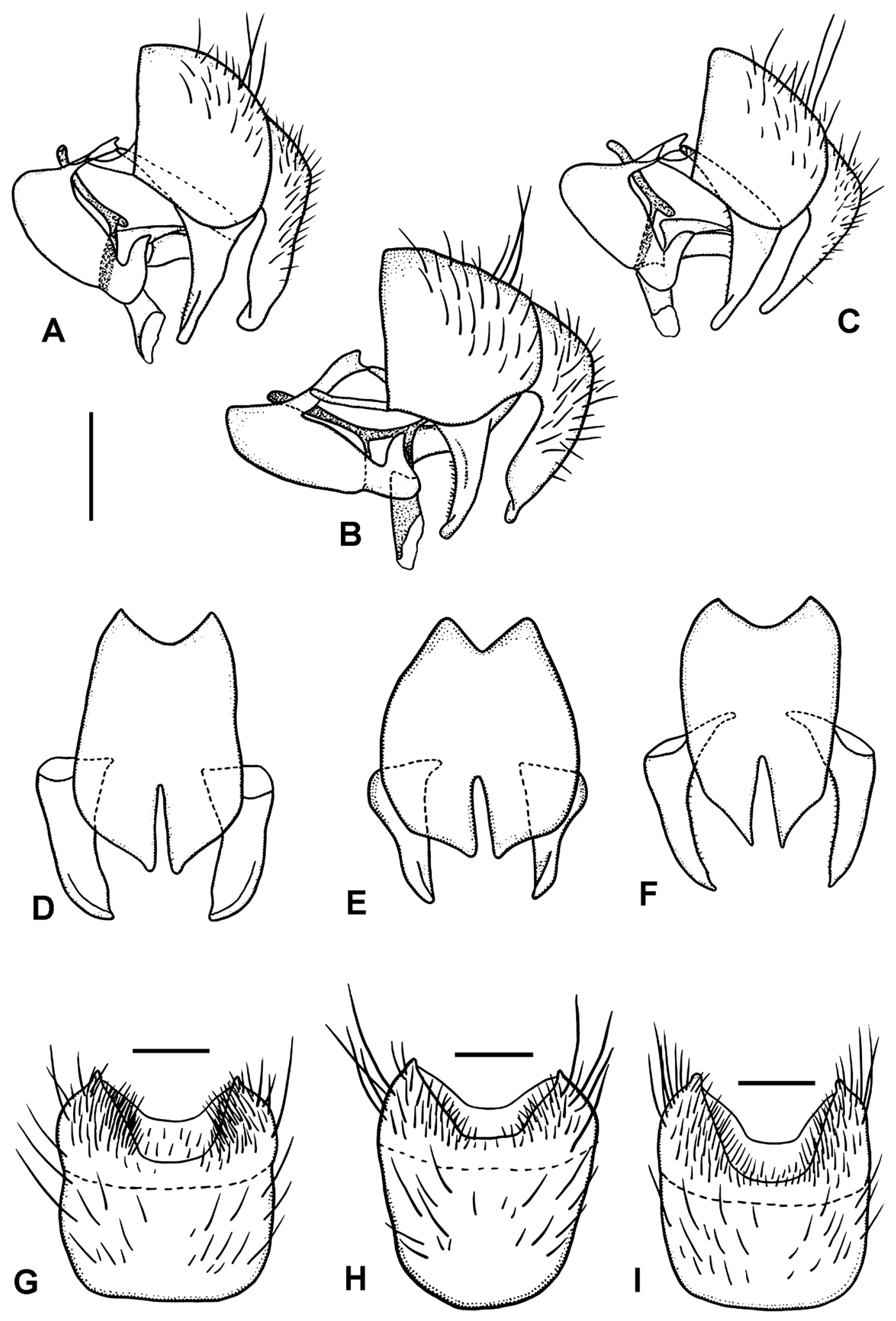

Fig. 5. Males of Spilogona Schnabl, 1911. A-C. Terminalia, lateral view. A. S. imitatrix (Malloch, 1921). B. S. nitidicauda (Schnabl, 1911). C. S. platyfrons Sorokina, 2018. D-F. Cercal plate, posterior view. D. S. imitatrix. E. S. nitidicauda. F. S. platyfrons. G-I. Sternite 5. G. S. imitatrix. H. S. nitidicauda. I. S. platyfrons. Scale bars: $0.25 \mathrm{~mm}$. 
Table 3. Diagnostic characters of the 'Spilogona nitidicauda species-group'.

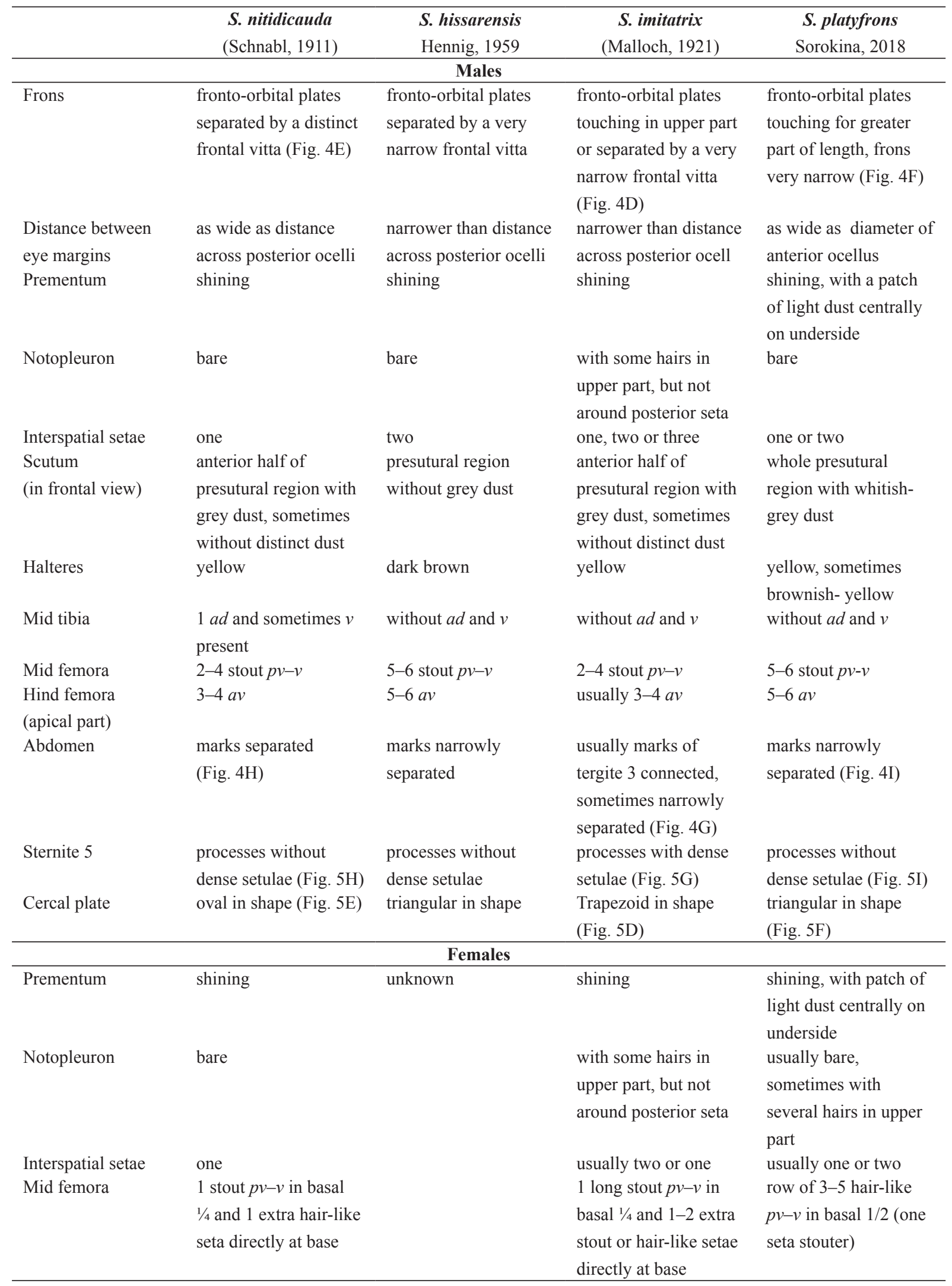


The species of this group can be separated by the following couples:

\section{Males}

1. In frontal view, presutural region of scutum without grey dust; haltere dark brown

S. hissarensis Hennig, 1959

- In frontal view, presutural region of scutum with grey dust; haltere yellow or brownish-yellow .... 2

2. Fronto-orbital plates touching along the greater part of their length, narrower than diameter of anterior ocellus; distance between eye margins on upper part of frons about as wide as diameter of anterior ocellus; prementum shining but with a patch of light dust centrally on underside; presutural region mostly with whitish-grey dust; mid femur with 5-6 long and stout $p v-v$ in basal half; hind femur with 5-6 av at apex

S. platyfrons Sorokina, 2018

- Fronto-orbital plates touching only in upper part or separated by a narrow frontal vitta; distance between eye margins on upper part of frons as wide as or narrower than the distance between posterior ocelli; prementum completely shining, without any dust; extreme anterior half of presutural region with grey dust; mid femur with 2-4 stout $p v-v$ in basal half; hind femur with 3-4 av at apex ....... 3

3. Notopleuron with some hairs in upper part, but not around posterior seta; fronto-orbital plates touching in upper part or separated by a very narrow frontal vitta; mid tibia without $a d$ and $v$; dark marks on tergite 3 usually connected or narrowly separated; caudal margin of sternite 5 with dense setulae

S. imitatrix (Malloch, 1921)

- Notopleuron completely bare; fronto-orbital plates usually separated by a distinct frontal vitta, sometimes touching; mid tibia with $1 \mathrm{ad}$ and sometimes a short $v$ present; tergites with well separated marks; caudal margin of sternite 5 without dense setulae

S. nitidicauda (Schnabl, 1911)

\section{Females}

1. Prementum of proboscis shining but with a patch of light dust centrally on underside; mid femur with a row of 3-5 hair-like $p v-v$ in basal $1 / 2$, sometimes one of them stouter

S. platyfrons Sorokina, 2018

- Prementum of proboscis completely shining; mid femur with 1 stout $p v-v$ in basal $1 / 4$ and 1-2 additional stout or hair-like setae directly at base

2. Notopleuron completely bare; anepisternum with 1 interspatial seta; mid tibia usually with $a d$; mid femur with 1 stout $p v-v$ in basal $1 / 4$ and 1 additional hair-like seta ......S. nitidicauda (Schnabl, 1911)

- Notopleuron with some hairs in upper part; usually anepisternum with 2 interspatial setae, rarely with one; mid tibia without ad; mid femur with 1 long stout $p v-v$ in basal $1 / 4$ and $1-2$ additional stout or hair-like setae

S. imitatrix (Malloch, 1921)

\section{Type locality}

Spilogona hissarensis Hennig, 1959

TAJIKISTAN: Varzob.

\section{Material examined}

\section{Holotype}

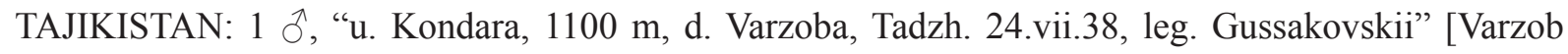

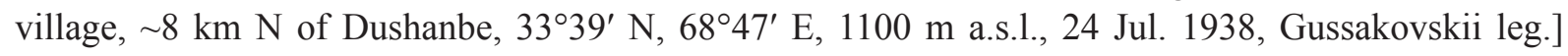
(ZISP). 


\section{Paratype}

TAJIKISTAN: 2 $\widehat{\jmath}$, “u. Kvak-Ruidasht, Gissar. khr. Tadzh., 16.vi.38., Gussakovskii” [Gissar Mountains, Rudaki district, 16 Jun. 1938, Gussakovskii leg.] (ZISP).

\section{Distribution}

Central Palaearctic: Tajikistan.

\section{Remark}

Mountain species, but probably living in the arctic tundra too.

\section{Spilogona imitatrix (Malloch, 1921)}

Figs 4A, D, G, 5A, D, G

\section{Type locality}

CANADA: Labrador, Nain.

\section{Material examined}

CANADA: 2 우, Manitoba, $24 \mathrm{~km}$ E of Churchill, Pistol range, $58^{\circ} 45^{\prime} \mathrm{N}, 93^{\circ} 56^{\prime} \mathrm{W}$, sweep in vegetation, 12 Jul. 2007, A. Renaud leg. (SZMN).

RUSSIA: 1 đ , Altai Republic, Kosh-Agach district, upper part of Naryn-Gol River, $49^{\circ} 49^{\prime} \mathrm{N}, 89^{\circ} 32^{\prime}$

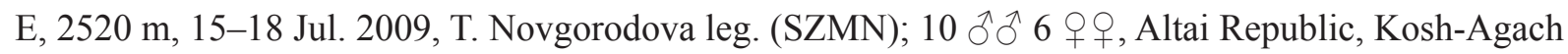
district, $7 \mathrm{~km} \mathrm{NW}$ of Kuray, south slope of Kurayskii mountain ridge, $50^{\circ} 18^{\prime} \mathrm{N}, 87^{\circ} 51^{\prime} \mathrm{E}, 2588 \mathrm{~m}$

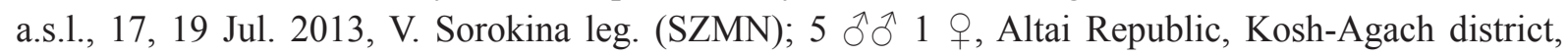
Ukok plateau, Rodonovyi spring, upper part of Zhumaly River, $49^{\circ} 27^{\prime} \mathrm{N}, 88^{\circ} 03^{\prime} \mathrm{E}, 2410 \mathrm{~m}$ a.s.1., 24

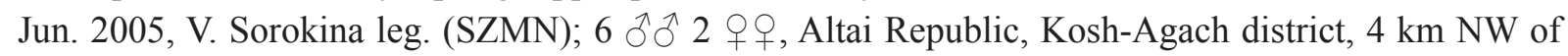

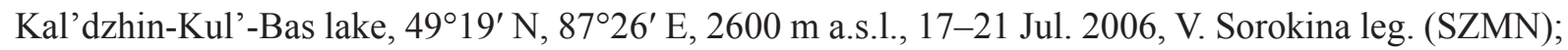

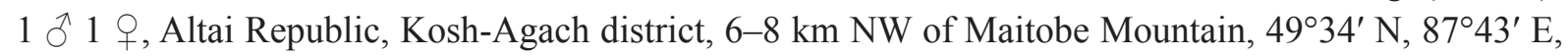

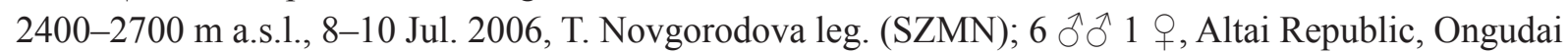
district, Terektinskiy range, upper part of Bolshoi Yaloman River, $50^{\circ} 28^{\prime} \mathrm{N}, 86^{\circ} 19^{\prime} \mathrm{E}, 2200-2300 \mathrm{~m}$ a.s.1., 3 Jul. 2007, V. Sorokina leg. (SZMN); 3 0 5 $q$, , Altai Republic, Ulagan district, environs of

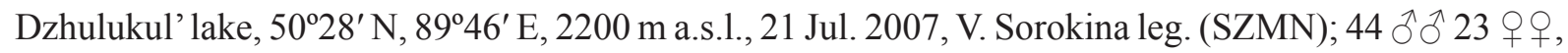
Altai Republic, Ulagan district, Shapshal'skiy mountain range, 50 $32^{\prime} \mathrm{N}, 89^{\circ} 48^{\prime} \mathrm{E}, 2550-2878 \mathrm{~m}$ a.s.l., on flowers of Schultzia crinita Spreng., 23-25 Jul. 2007, V. Sorokina and A. Barkalov leg. (SZMN); 1

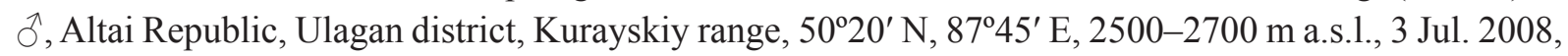
A. Barkalov leg. (SZMN).

\section{Distribution}

Holarctic Region; in the Palaearctic only from southern Siberia. Arcto-alpine species.

Spilogona nitidicauda (Schnabl, 1911)

Figs 4B, E, H, 5B, E, H

\section{Type locality}

RUSSIA: Yamalo-Nenetsk AO. 


\section{Material examined}

\section{Syntype}

RUSSIA: 5 万ิ 5 우, “Karskaja tundra, s. Tobol's. gub., 21.vii.09, Zaizev” [Yamalo-Nenetskiy AO, east slope of Polar Ural, mouth of the River Kara, $\sim 69^{\circ} 02^{\prime} \mathrm{N}, 64^{\circ} 35^{\prime} \mathrm{E}, 21$ Jul. 1909, Zaizev leg.]. 1 oे, holotype of Limnophora (Spilogona) nitidicauda var. depressa Schnabl, same data as for preceding (ZISP).

\section{Other material examined}

RUSSIA: 9 ๙ึ 2 우, Altai Republic, Kosh-Agach district, Ukok plateau, environs of Muzdy-Bulak lake, $49^{\circ} 17^{\prime}$ N, $87^{\circ} 39^{\prime}$ E, 2450 m a.s.1., 26 Jun. 2005, 1-2 Jul. 2005, A. Barkalov and V. Sorokina leg. (SZMN); 24 ठิ 8 우, Altai Republic, Kosh-Agach district, Ukok plateau, environs of Kal'dzhin-Kul'Bas lake, 49 $19^{\prime} \mathrm{N}, 8^{\circ} 26^{\prime}$ E, 2400-2600 m a.s.1., 17-21 Jul. 2006, V. Sorokina and T. Novgorodova

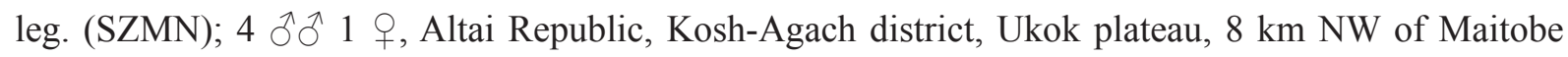

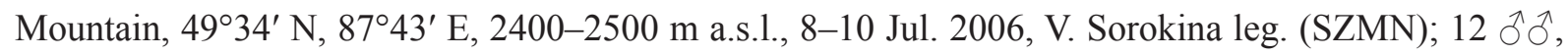
Altai Republic, Kosh-Agach district, Ukok plateau, Rodonovyi spring, upper part of Zhumaly River,

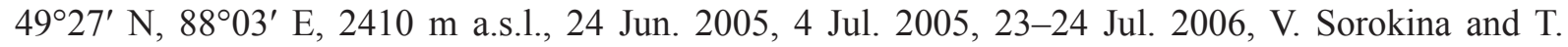
Novgorodova leg. (SZMN); 1 § 1 \&, Altai Republic, Kosh-Agach district, upper part of Naryn-Gol River, 4949' N, 89³2' E, 2520 m a.s.1., 15-19 Jul. 2009, V. Sorokina and T. Novgorodova leg. (SZMN); $1{ }^{\lambda}$, Altai Republic, Kosh-Agach district, Severo-Chuyskyi Ridge, valley of Aktru River, $50^{\circ} 06^{\prime} \mathrm{N}$, 8748' E, 1865-2064 m a.s.1., 21 Jul. 2013, T. Novgorodova leg. (SZMN); 2 우으, Altai Republic, Ongudai district, Terektinskiy range, upper part of Bolshoi Yaloman River, 50 $28^{\prime}$ N, 86 $16^{\prime}$ E, 2200 2300 m a.s.l., 3 Jul. 2007, V. Sorokina leg. (SZMN); 1 o, Altai Republic, Ulagan district, Kurayskiy

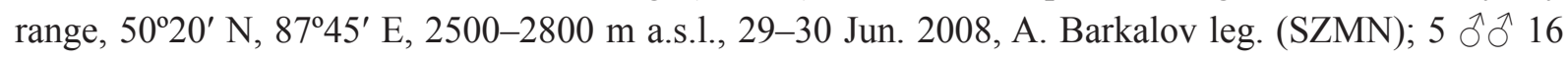
우, Altai Republic, Ulagan district, environs of Dzhulukul' lake, 50 $28^{\prime} \mathrm{N}, 89^{\circ} 46^{\prime} \mathrm{E}, 2200 \mathrm{~m}$ a.s.l., 21 Jul. 2007, V. Sorokina leg. (SZMN); 2 ㅈํ 5 우우, Altai Republic, Ulagan district, Shapshal'skiy mountain range, upper part of Shuy River, $50^{\circ} 32^{\prime} \mathrm{N}, 8^{\circ} 48^{\prime}$ E, 2550-2878 m a.s.1., 23-25 Jul. 2007, V. Sorokina leg. (SZMN); 1 \ 1 + , Altai Republic, Ust-Koksa district, 47 km W Ust-Koksa, Krasnaya

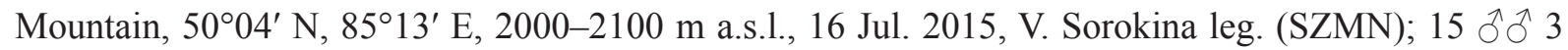
우, Chukotka AO, Beringovskii district, $40 \mathrm{~km}$ SSW of Beringovskii, 62 $43^{\prime} \mathrm{N}, 178^{\circ} 55^{\prime}$ E, yellow plate trap, 22-23, 26 Jul. 2012, A. Stekolshchikov leg. (SZMN); 1 q, Chukotka AO, $30 \mathrm{~km} \mathrm{NW}$ of

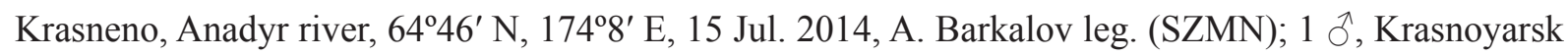
Kray, Taymyr Peninsula, Kuria village, upper part of Pyasina river $\left[70^{\circ} 16^{\prime} \mathrm{N}, 88^{\circ} 30^{\prime}\right.$ E], alder forest near village, 13 Jul. 1967, K. Gorodkov leg. (ZISP); 1 \&, Krasnoyarsk Kray, Taymyr Peninsula, 114 km S of Khatanga, Kotuy river, $71^{\circ} 02^{\prime} \mathrm{N}, 103^{\circ} 00^{\prime} \mathrm{E}, 2-5$ Jul. 2010, A. Barkalov leg. (SZMN); 1 ` 2 우우,

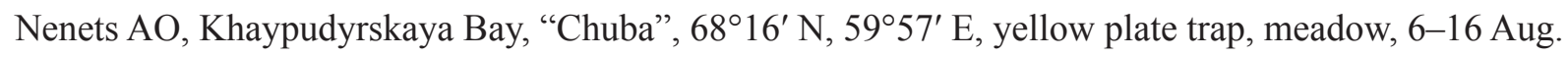

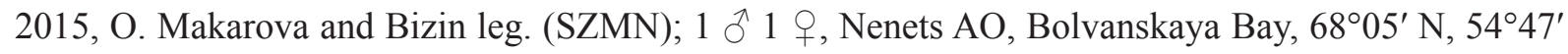
E, yellow plate trap, anthropogenic meadow, 18-25 Jul. 2015, O. Makarova and Bizin leg. (SZMN); 1

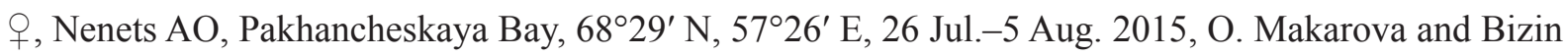
leg. (SZMN); $2 \partial^{\curvearrowright}$, Yamalo-Nenets AO, $73 \mathrm{~km} \mathrm{NE}$ of Labytnangi, Longot'egan river, $67^{\circ} 19^{\prime} \mathrm{N}, 66^{\circ} 43^{\prime}$ E, 175 m a.s.1., 2, 10 Jul. 2015, A. Barkalov leg. (SZMN).

SWEDEN: 1 đ̊, Abisko, Nuolja, meadow below Topstation, 19 Jul. 1987, A. Pont leg. (SZMN).

\section{Distribution}

Holarctic Region; in the Palaearctic, from North Scandinavia, Russia and China. Arcto-alpine species. 


\section{Spilogona platyfrons Sorokina, 2018}

Figs 4C, F, I, 5C, F, I

\section{Type locality}

RUSSIA: Altai Mountains.

\section{Material examined}

\section{Holotype}

RUSSIA: 1 ${ }^{7}$, Altai Republic, Ulagan district, Shapshal'skiy mountain range, upper part of Shui River, $\sim 50^{\circ} 33^{\prime} \mathrm{N}, 89^{\circ} 50^{\prime}$ E, 2550-2900 m a.s.1., 25 Jul. 2007, V. Sorokina leg. (SZMN).

\section{Paratypes}

RUSSIA: 34 कิ 21 우, Altai Republic, Ulagan district, Shapshal'skiy mountain range, upper part of Shui River, $\sim 50^{\circ} 33^{\prime}$ N, 89 $50^{\prime}$ E, 2550-2900 m a.s.1., 25 Jul. 2007, V. Sorokina leg. (2 males and 2 females in ZMUM \& ZISP, the rest in SZMN); 1 , Altai Republic, Kosh-Agach district, upper part of

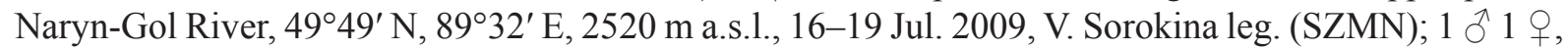
Altai Republic, Kosh-Agach district, Ukok plateau, environs of Muzdy-Bulak lake, 49 $17^{\prime}$ N, $87^{\circ} 39^{\prime}$ E,

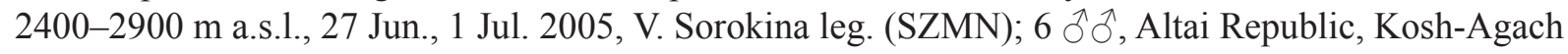

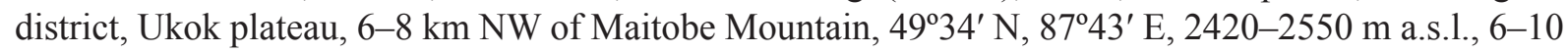
Jul. 2006, V. Sorokina and T. Novgorodova leg. (SZMN); 6 주 4 우우, Altai Republic, Kosh-Agach district, Ukok plateau, environs of Kal'dzhin-Kul'-Bas lake, 49 ${ }^{\circ} 19^{\prime}$ N, $87^{\circ} 26^{\prime}$ E, 2400-2600 m a.s.1., 18, 20-21 Jul. 2006, V. Sorokina and T. Novgorodova leg. (SZMN); 17 ๙ $\widehat{\jmath} 3$ 우우, Altai Republic, KoshAgach district, Ukok plateau, south slope of Yuzhno-Chuiskiy range, valley of Tara River, $49^{\circ} 39^{\prime} \mathrm{N}$,

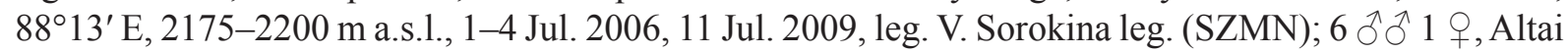
Republic, Kosh-Agach district, Ukok plateau, upper part of Zhumaly river, Rodonovyi spring, $49^{\circ} 16^{\prime}$

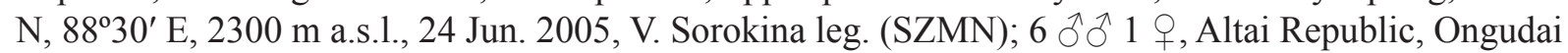
district, Terektinskiy range, upper part of Bolshoi Yaloman River, 2200-2300 m a.s.1., 3 Aug. 2007, V. Sorokina leg. (SZMN); 1 ${ }^{\prime}$, Altai Republic, Ulagan district, Kurayskiy range, 50 $20^{\prime} \mathrm{N}, 87^{\circ} 45^{\prime} \mathrm{E}$, 2500-2800 m a.s.1., 29-30 Jun. 2008, A. Barkalov leg. (SZMN); 13 đ઼ 20 우우, Altai Republic, Ulagan district, environs of Dzhulukul' lake, 50²8' N, 89²46' E, 2200 m a.s.l., 21-24 Jul. 2007, V. Sorokina leg. (SZMN); 18 万ð 3 우오. Altai Republic, Ust-Koksa district, $47 \mathrm{~km} \mathrm{~W}$ of Ust-Koksa, Krasnaya Mountain,

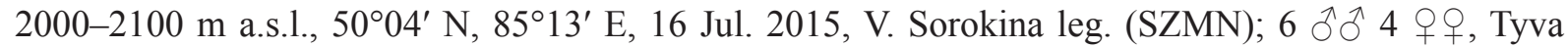
Republic, Morgun-Taiginskii kozhuun, environs of Mugur-Aksy, $\sim 50^{\circ} 22^{\prime} \mathrm{N}, 9^{\circ} 25^{\prime} \mathrm{E}, 2000-2100 \mathrm{~m}$ a.s.1., 22 Jul. 1993, A. Barkalov leg. (SZMN).

\section{Distribution}

Palaearctic Region: southern Siberia.

\section{Remark}

Mountain species, but probably living in the arctic tundra too.

\section{Molecular-genetic analysis of 'Spilogona nitidicauda species-group'}

Since only the old type specimens of $S$. hissarensis are known, the molecular analysis was performed on the other three species of this group.

DNA variability is higher within taxa of the 'Spilogona nitidicauda species-group' in comparison with taxa of the 'Spilogona contractifrons species-group'. Intraspecies DNA variability was found in S. nitidicauda and S. platyfrons from different localities. The isolate SN38 of Spilogona nitidicauda from the Altai (Muzdy-Bulak lake) differs from the isolates SN40 and SN41 of S. nitidicauda from 
Chukotka AO (Beringovskii) by a synonymous substitution of C186T. Both isolates from Chukotka have identical DNA. The SP32 of S. platyfrons from the Shapshalsky ridge (Altai) differs from the SP33 of $S$. platyfrons from the Krasnaya Mountain (Altai) by substitutions of T636C (Supplementary file). Spilogona nitidicauda can be distinguished from S. platyfrons by 3-4 SNPs. Spilogona imitatrix differs from $S$. platyfrons with $6 \mathrm{SNP}$, and from $S$. nitidicauda with 7 SNP.

The genetic distance between $S$. nitidicauda and $S$. platyfrons is $0.4 \%$, between $S$. platyfrons and S. imitatrix is $1.2 \%$, and between $S$. nitidicauda and S. imitatrix is $1.6 \%$. All three species formed distinct clusters on the ML tree, but the differences between $S$. nitidicauda and S. platyfrons are insignificant statistically.

The following results were obtained after comparing the sequences of the studied specimens with the annotated sequences in GenBank. Unfortunately, there were no DNA sequences of $S$. nitidicauda and S. platyfrons in GenBank nor in the BOLD database, so comparison with our results was impossible. But there were many DNA sequences of $S$. imitatrix from Canada.

Spilogona imitatrix from the Altai is identical with several individuals of S. imitatrix from Canada (KR615453 and KR622696). However, several individuals from GenBank identified as S. imitatrix have a very variable DNA, as can be seen on the ML tree (Fig. 3). Such a high polymorphism may be due to errors in morphological identification.

\section{Discussion}

The analysis of genetic distances based on the differences in the cytochrome oxidase I gene in members of the 'Spilogona contractifrons species-group' and the 'Spilogona nitidicauda species-group' confirmed that there are not only morphological differences but also genetic differences between closely related species.

Our results with the DNA analysis of the COI gene have demonstrated that S. alticola, S. contractifrons and $S$. arctica are not synonymous because the genetic differences between individuals of these species persist in geographically distant locations (Fig. 6). The DNA of S. arctica is identical in individuals from different regions of Russia, Sweden, Greenland and Canada. Individuals of $S$. contractifrons from Russia are identical with $S$. contractifrons from Sweden and Norway, and all of them form a distinct cluster of "European" or "Palaearctic" S. contractifrons. It should be emphasised that the specimens of $S$. arctica and $S$. contractifrons from Russia were identical with specimens from the type localities (Sweden and Greenland) but show differences from specimens of these species from Canada.

In turn, specimens from Canada designated in GenBank as $S$. contractifrons were identical with specimens from the Altai identified by us as S. alticola. The latter species was described from specimens from USA (New Hampshire) and was later found in different areas of Northern Canada (Huckett 1965). The reasons for this result could be either the incorrect identification of the specimens deposited in GenBank, or a rejection of the existence of $S$. alticola species by Canadian researchers and a designation of such specimens as $S$. contractifrons.

In the 'Spilogona nitidicauda species-group' there are stable nucleotide differences between S. nitidicauda and $S$. platyfrons from different places and years of collection despite the morphological similarity. Although the genetic distance between $S$. nitidicauda and $S$. platyfrons is only $0.4 \%, S$. imitatrix differs from them significantly. The DNA distance between $S$. imitatrix and $S$. nitidicauda is $1.6 \%$, and that between $S$. imitatrix and S. platyfrons is $1.2 \%$ which demonstrates the separate species status of S. imitatrix. In Muscidae, ranges of $0.18 \%$ and $3.01 \%$ have been reported as averages for the means and maxima of COI intraspecific distances, respectively (Renaud et al. 2012). Limits of species with a 
distance to the nearest neighbour of $<2 \%$ in 137 species of Muscidae were supported by morphological characters and confirmed that the minimum interspecific distance in Muscidae ranges well below $2 \%$ for many species (Renaud et al. 2012).

Spilogona imitatrix was described from specimens from Labrador (Canada) and was later found in different regions of Canada and USA (Alaska). Recently this species was found in the Palaearctic Region for the first time, here known only in the Altai Mountains (Sorokina 2018). The DNA of S. imitatrix from the Altai (Terektinsky ridge) was identical with the DNA of several individuals of $S$. imitatrix from the Northwest Territories (Canada). There is a rather large number of the sequences of this species
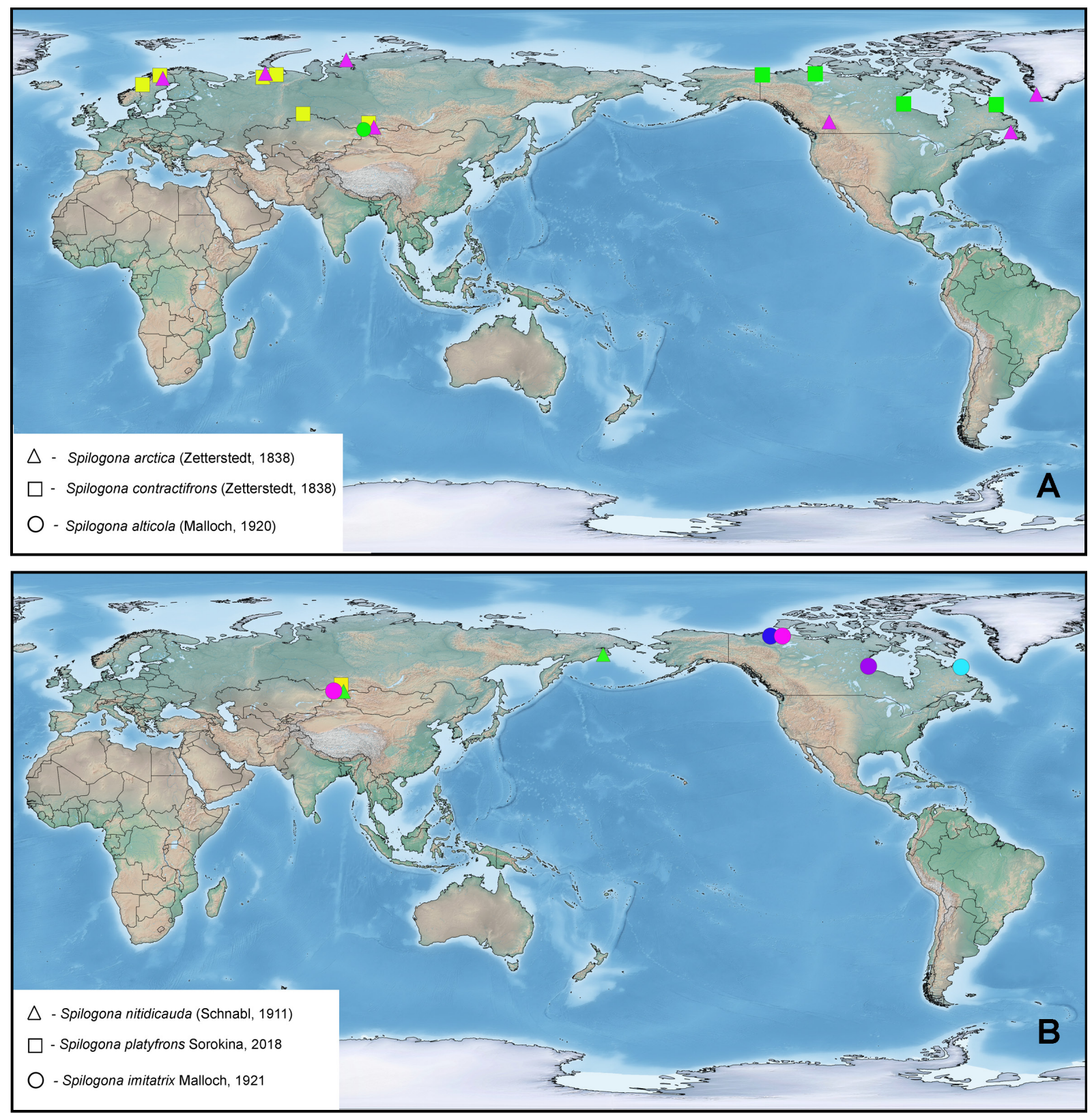

Fig. 6. Localities of the species of Spilogona Schnabl, 1911 used in the DNA analysis. Symbols denote the species, whilst the colour shows the same DNA sequences. A. 'S. contractifrons species-group'. B. 'S. nitidicauda species-group'. 
from Canada in GenBank. Several DNA sequences of S. imitatrix from Canada were randomly taken for genetic matching with the specimen of this species from the Altai. It transpired that one sequence (KR628150) clustered with S. platyfrons from the Altai, whilst the other sequences of Canadian specimens (\# HM389123, \# HM389124) differed from all of them by six nucleotide substitutions (Fig. 3). Such differences between DNA sequences of one species can be explained by errors in the initial morphological identification. This has been proved by the work of the first author of this paper (VSS) with the Muscidae collection in the CNC, where the following species were found under the name S. imitatrix, and vice versa: S. alticola, S. arctica, S. deflorata (Holmgren, 1872), S. incauta (Huckett, 1932), S. nitidicauda, S. trigonifera (Zetterstedt, 1838), S. sororcula (Zetterstedt, 1845), S. trilineata (Huckett, 1932) and another Spilogona sp.

These misidentifications are not surprising, since most members of the genus Spilogona are difficult to distinguish, even for muscid specialists. Therefore, in addition to a genetic analysis it is important to have an idea of the most stable morphological characters in each group. According to our results with this genus, the following can be considered as the most stable morphological characters in Spilogona: presence of $p v / v$ setae on mid tibia, presence of $p v$ setae on hind femur, presence of dust on the prementum, shape of the sternite 5 , presence interspatial seta(e) on anepisternum, number of post $d c$ setae on the scutum (3 or 4), presence on the scutellum of downwardly-directed preapical setulae on upper border of declivities. The last character was proposed and used only by Huckett (1965) for the separation of species into groups in his key to the species of Spilogona, in addition to the other well-known characters. Other researchers (Hennig 1959a, 1959b, 1959c; Gregor et al. 2002) used well-established characters for identification, specifically the number of post $d c$ setae on the scutum (3 or 4), the elongation of the face, and the presence of $p v$ setae on hind femur. The character suggested by Huckett (downwardlydirected preapical setulae on the upper border of declivities of scutellum) is quite difficult to see, which is probably why it has not been generally used by the authors of the keys. However, in our opinion, this is one of the important characters for the identification of the species of Spilogona. To demonstrate this, several outgroup species (S. aerea (Fallén, 1825), S. bifimbriata Huckett, 1965, S. baltica (Ringdahl, 1918) and S. tornensis (Ringdahl, 1926)) were included in the genetic analysis. These species have the same characters ( 3 and 4 post $d c$ setae, mid tibia without $v$ seta, hind femur with and without $p v$ setae, face not projecting) as in the species groups analysed above, but, unlike them, these species have scutellum with downwardly-directed preapical setulae on the upper border of declivities. The results of the genetic analysis showed a distinct distance between the cluster of the 'Spilogona contractifrons species-group' and the 'Spilogona nitidicauda species-group' on the one hand, the members of which have scutellum without downwardly-directed preapical setulae on the upper border of declivities, and on the other hand the cluster of species having these setulae on the scutellum (Fig. 3).

The members of the 'Spilogona contractifrons species-group' and the 'Spilogona nitidicauda speciesgroup' thus have not only distinguishing morphological characters but also fixed substitutions in the DNA sequences. Since a low interspecific polymorphism is known in the Muscidae, the revealed genetic distances confirm the existence of separate species or subspecies in each of the groups studied.

\section{Acknowledgments}

We would especially like to thank Adrian Pont (Oxford, UK) for discussion of this manuscript and for checking the English. The research presented in this paper was supported by the Federal Fundamental Scientific Research Programme for 2013-2020, the projectNo.VI.51.1.5(AAAA-A16-116121410121-7) and the Russian Federation Budget Project No. AAAA-A16-116111610180-3. 


\section{References}

Collin J.E. 1930. A revision of the Greenland species of the anthomyid genus Limnophora sens. lat. (Diptera), with figures of the male genitalia of these and many other Palaearctic species. Transactions of the Entomological Society of London 78: 255-281. https://doi.org/10.1111/j.1365-2311.1930.tb00387.x

Gregor F., Rozkošný R., Barták M. \& Vaňhara J. 2002. The Muscidae (Diptera) of Central Europe. Folia Facultatis Scientiarum Naturalium Universitatis Masarykianae Brunensis, Biologia 107: 1-280.

Haseyama K.L.F., Wiegmann B.M., Almeida E.A.B. \& de Carvalho C.J.B. 2015. Say goodbye to tribes in the new house fly classification: a new molecular phylogenetic analysis and an updated biogeographical narrative for the Muscidae (Diptera). Molecular Phylogenetics and Evolution 89: 1-12. https://doi.org/10.1016/j.ympev.2015.04.006

Hennig W. 1959a. Muscidae [Part, Lieferung 204]. In: Lindner E. (ed.) Die Fliegen der palaearktischen Region 63b: 233-288. Schweizerbart, Stuttgart.

Hennig W. 1959b. Muscidae [Part, Lieferung 205]. In: Lindner E. (ed.) Die Fliegen der palaearktischen Region 63b: 289-336. Schweizerbart, Stuttgart.

Hennig W. 1959c. Muscidae [Part, Lieferung 207]. In: Lindner E. (ed.) Die Fliegen der palaearktischen Region 63b: 337-384. Schweizerbart, Stuttgart.

Huckett H.C. 1965. The Muscidae of Northern Canada, Alaska and Greenland (Diptera). Memoirs of the Entomological Society of Canada 42: 5-369. https://doi.org/10.4039/entm9742fv

Kutty S.N., Pape T., Pont A.C., Wiegmann B.M. \& Meier R. 2008. The Muscoidea (Diptera: Calyptratae) are paraphyletic: evidence from four mitochondrial and four nuclear genes. Molecular Phylogenetics and Evolution 49: 639-652. https://doi.org/10.1016/j.ympev.2008.08.012

Kutty S.N., Pont A.C., Meier R. \& Pape T. 2014. Complete tribal sampling reveals basal split in Muscidae (Diptera), confirms saprophagy as ancestral feeding mode, and reveals an evolutionary correlation between instar numbers and carnivory. Molecular Phylogenetics and Evolution 78: 349-364. https://doi.org/10.1016/j.ympev.2014.05.027

Michelsen V. 2006. Annotated catalogue of the Anthomyiidae, Fanniidae, Muscidae and Scathophagidae (Diptera: Muscoidea) of Greenland. Steenstrupia 29: 105-126.

Pont A.C. 1986. Family Muscidae. In: Soós Á. \& Papp L. (eds) Catalogue of Palaearctic Diptera 11: 57-215. Akadémiai Kiadó, Budapest.

Ratnasingham S. \& Hebert P.D.N. 2007. BOLD: The Barcode of Life Data System (www.barcodinglife. org). Molecular Ecology Notes 7: 355-364. https://doi.org/10.1111/j.1471-8286.2006.01678.x

Renaud A.K., Savage J. \& Adamowicz S.J. 2012. DNA barcoding of Northern Nearctic Muscidae (Diptera) reveals high correspondence between morphological and molecular species limits. $B M C$ Ecology 12 (1): 1-15. https://doi.org/10.1186/1472-6785-12-24

Savage J., Wheeler T.A. \& Wiegmann B.M. 2004. Phylogenetic analysis of the genus Thricops Rondani (Diptera: Muscidae) based on molecular and morphological characters. Systematic Entomology 29: 395-414. https://doi.org/10.1111/j.0307-6970.2004.00252.x

Schuehli G.S.E., de Carvalho C.J.B. \& Wiegmann B.M. 2004. Regarding the taxonomic status of Ophyra Robineau-Desvoidy (Diptera: Muscidae): a molecular approach. Zootaxa 712: 1-12.

Schuehli G.S.E, de Carvalho C.J.B. \& Wiegmann BM. 2007. Molecular phylogenetics of the Muscidae (Diptera: Calyptratae): new ideas in a congruence context. Invertebrate Systematics 21: 263-278. https://doi.org/10.1071/IS06026 
Shaikevich E.V. 2007. PCR-RFLP of the COI gene reliably differentiates Cx. pipiens, Cx. pipiens form molestus and Cx. torrentium of the pipiens Complex. European Mosquito Bulletin 23: 25-30.

Simon C., Frati F., Beckenbach A., Crespi B., Liu H. \& Flook P. 1994. Evolution, weighting, and phylogenetic utility of mitochondrial gene sequences and a compilation of conserved polymerase chain reaction primers. Annals of the Entomological Society of America 87 (6): 651-701. https://doi.org/10.1093/aesa/87.6.651

Sorokina V.S. 2012a. Fauna of Muscidae (Diptera) of the Altai Mountains. Proceedings of the Russian Entomological Society 83 (1): 193-222. [In Russian, with English abstract.]

Sorokina V.S. 2012b. The Muscidae (Diptera) of the Russian tundra zones. Report 1. Caucasian Entomological Bulletin 8 (2): 328-332. [In Russian, with English abstract.]

Sorokina V.S. 2013a. Fauno-genetic connections of Muscoidea (Diptera) of the high altitude of the Altai Mts and the latitudinal tundra of Taymyr Peninsula. In: Babin V.G., Tabakaev U.V., Dolgovykh S.V., Yurkova N.A., Aleynikova V.N. \& Bondarenko A.V., Biodiversity, ecological issues of Gorny Altai and its neighboring regions: present, past and future: 115-118. Russia, Gorno-Altaisk. [In Russian, with English summary.]

Sorokina V.S. 2013b. Muscidae from above the tree-line in the Altai Mountains. In: Babin V.G., Tabakaev U.V., Dolgovykh S.V., Yurkova N.A., Aleynikova V.N. \& Bondarenko A.V., Biodiversity, ecological issues of Gorny Altai and its neighboring regions: present, past and future: 118-122. Russia, GornoAltaisk. [In Russian, with English summary.]

Sorokina V.S. 2017. The Muscoidea Flies (Diptera) of the northern territories of Russia. Euroasian Entomological Journal 16(1): 44-56. [In Russian, with English summary.]

Sorokina V.S. 2018. Eleven new species of Spilogona Schnabl, 1911 (Diptera, Muscidae) from the Altai Mountains of Russia, with key to species. Zootaxa 4410 (2): 201-250. https://doi.org/10.11646/zootaxa.4410.2.1

Sorokina V.S. \& Khruleva O.A. 2012. Details of species composition and distribution of house-flies (Diptera, Muscidae) of the Wrangel Island, Russia. Euroasian Entomological Journal 11: 553-564. [In Russian with English abstract.]

Tamura K., Stecher G., Peterson D., Filipski A. \& Kumar S. 2013. MEGA6: Molecular Evolutionary Genetics Analysis version 6.0. Molecular Biology and Evolution 30 (12): 2725-2729. https://doi.org/10.1093/molbev/mst197

Werner D. \& Pont A.C. 2006a. The feeding and reproductive behavior of the Limnophorini (Diptera: Muscidae). Studia Dipterologica, Supplement, Halle/Saale 14: 79-114.

Werner D. \& Pont A.C. 2006b. New results on Diptera predators in the blackfly plague areas of Central Europe and the Caucasus. Acta Entomologica Serbica, Supplement: 131-140. 
Manuscript received: 13 September 2018

Manuscript accepted: 15 October 2018

Published on: 11 December 2018

Topic editor: Gavin Broad

Desk editor: Alejandro Quintanar

Printed versions of all papers are also deposited in the libraries of the institutes that are members of the EJT consortium: Muséum national d'Histoire naturelle, Paris, France; Meise Botanic Garden, Belgium; Royal Museum for Central Africa, Tervuren, Belgium; Natural History Museum, London, United Kingdom; Royal Belgian Institute of Natural Sciences, Brussels, Belgium; Natural History Museum of Denmark, Copenhagen, Denmark; Naturalis Biodiversity Center, Leiden, the Netherlands; Museo Nacional de Ciencias Naturales-CSIC, Madrid, Spain; Real Jardín Botánico de Madrid CSIC, Madrid, Spain; Zoological Research Museum Alexander Koenig, Bonn, Germany. 